\title{
Calibration and Limitations of the Mg II Line-based Black Hole Masses
}

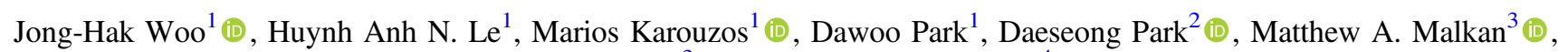 \\ Tommaso Treu $^{3}$ (D), and Vardha N. Bennert ${ }^{4}$ (D) \\ ${ }^{1}$ Astronomy Program, Department of Physics and Astronomy, Seoul National University, Seoul, 08826, Republic of Korea \\ ${ }^{2}$ Korea Astronomy and Space science Institute, Daejeon, Republic of Korea \\ ${ }^{3}$ Department of Physics and Astronomy, University of California, Los Angeles, CA 90095, USA \\ ${ }^{4}$ Physics Department, California Polytechnic State University, San Luis Obispo, CA 93407, USA \\ Received 2017 November 26; revised 2018 April 7; accepted 2018 April 16; published 2018 June 1
}

\begin{abstract}
We present single-epoch black hole mass $\left(M_{\mathrm{BH}}\right)$ calibrations based on the rest-frame ultraviolet (UV) and optical measurements of Mg II $2798 \AA$ and $\mathrm{H} \beta 4861 \AA$ lines and the active galactic nucleus (AGN) continuum, using a sample of 52 moderate-luminosity AGNs at $z \sim 0.4$ and $z \sim 0.6$ with high-quality Keck spectra. We combine this sample with a large number of luminous AGNs from the Sloan Digital Sky Survey to increase the dynamic range for a better comparison of UV and optical velocity and luminosity measurements. With respect to the reference $M_{\mathrm{BH}}$ based on the line dispersion of $\mathrm{H} \beta$ and continuum luminosity at $5100 \AA$, we calibrate the UV and optical mass estimators by determining the best-fit values of the coefficients in the mass equation. By investigating whether the UV estimators show a systematic trend with Eddington ratio, FWHM of $\mathrm{H} \beta, \mathrm{Fe}$ II strength, or UV/optical slope, we find no significant bias except for the slope. By fitting the systematic difference of $\mathrm{Mg}$ II-based and $\mathrm{H} \beta$-based masses with the $L_{3000} / L_{5100}$ ratio, we provide a correction term as a function of the spectral index as $\Delta C=0.24$ $\left(1+\alpha_{\lambda}\right)+0.17$, which can be added to the Mg II-based mass estimators if the spectral slope can be well determined. The derived UV mass estimators typically show $>\sim 0.2$ dex intrinsic scatter with respect to the $\mathrm{H} \beta$-based $M_{\mathrm{BH}}$, suggesting that the UV-based mass has an additional uncertainty of $\sim 0.2$ dex, even if high-quality rest-frame UV spectra are available.
\end{abstract}

Key words: galaxies: active - galaxies: nuclei - galaxies: Seyfert - quasars: emission lines - quasars: supermassive black holes

\section{Introduction}

One of the most fundamental properties of active galactic nuclei (AGNs) is black hole $(\mathrm{BH})$ mass $\left(M_{\mathrm{BH}}\right)$, which sets the upper limit of AGN energetics via the Eddington limit. $M_{\mathrm{BH}}$ also represents the integration of mass accretion over the lifetime of a given $\mathrm{BH}$, connecting the growth histories of galaxies and $\mathrm{BHs}$ as implied by the observed correlation between $M_{\mathrm{BH}}$ and host galaxy properties (e.g., Kormendy \& Ho 2013; Woo et al. 2013).

Estimating $M_{\mathrm{BH}}$ became a routine process for type $1 \mathrm{AGNs}$, which are characterized by the presence of broad emission lines, since various single-epoch mass estimators were developed based on the empirical results from reverberationmapping studies. While the size of the broad-line region (BLR) is measured from the time lag between the light curves of the AGN continuum and broad emission line flux in the reverberation-mapping studies (Blandford \& McKee 1982; Peterson 1993), which requires a long-term monitoring campaign (e.g., Wandel et al. 1999; Kaspi et al. 2000; Peterson et al. 2004; Bentz et al. 2009; Barth et al. 2011, 2015; Grier et al. 2013; Fausnaugh 2017; Park et al. 2017), single-epoch estimators utilize the empirical size-luminosity relation obtained from the reverberation studies. As a proxy for the BLR size, the monochromatic luminosity at $5100 \mathrm{~A}$ can be used to indirectly infer the BLR size based on the empirical relation (e.g., Kaspi et al. 2000, 2005; Bentz et al. 2006, 2013).

Since the size-luminosity relation provides a powerful simple method for determining $M_{\mathrm{BH}}$, which only requires single spectroscopic observation, it has been applied to a large sample of type 1 AGNs (e.g., Woo \& Urry 2002; McLure \& Dunlop 2004; Shen et al. 2011). While the $\mathrm{H} \beta$ line was the main tool to measure the time lag in the reverberation-mapping studies, so the size-luminosity relation is best-calibrated with the $\mathrm{H} \beta$ line, the single-epoch method with various recipes became applicable to type 1 AGNs at higher redshift. In this case, the rest-frame UV continuum and emission lines obtained in large optical spectroscopic surveys, e.g., the Sloan Digital Sky Survey (SDSS), are typically used to estimate $M_{\mathrm{BH}}$. For example, a combination of the Mg II $2798 \AA$ line velocity and near-ultraviolet (near-UV) continuum luminosity has been used for AGNs at $0.4<z<2$ (e.g., McLure \& Dunlop 2004; Vestergaard \& Peterson 2006; McGill et al. 2008; Wang et al. 2009; Shen et al. 2011), while the combination of the velocity of the C IV $1549 \AA$ line and AGN contiuum luminosity in the far-UV is often adopted for AGNs at $z>\sim 2$ (e.g., Shen et al. 2011; Karouzos et al. 2015).

By combining the virial assumption, i.e., the BLR gas is mainly governed by the gravitational potential of the central $\mathrm{BH}$, and the size-luminosity relation between BLR size $\left(R_{\mathrm{BLR}}\right)$ and continuum luminosity $(L)$ as $R_{\mathrm{BLR}} \propto \mathrm{L}^{\sim 0.5}, M_{\mathrm{BH}}$ can be expressed as

$$
\log M_{\mathrm{BH}}=\alpha+\beta \log V+\gamma \log L
$$

where $V$ is the velocity measured from the width of a broad emission line. For $\mathrm{H} \beta$-based mass estimators, $\beta$ is fixed as 2 , based on the virial theorem. However it can differ from this as the comparison between $\mathrm{H} \beta$ line width and that of other broad emission lines often shows a nonlinear relationship (e.g., Wang et al. 2009; Marziani et al. 2013). In the case of $\gamma$, the most updated $\mathrm{H} \beta$ size-luminosity relation study reported $\gamma=$ $0.533_{-0.033}^{0.035}$ (Bentz et al. 2013), which is consistent with a naive 
Table 1

Target Properties

\begin{tabular}{|c|c|c|c|c|c|c|c|c|c|c|c|c|c|c|}
\hline $\begin{array}{l}\text { Name } \\
\text { (1) }\end{array}$ & $\begin{array}{c}z \\
(2)\end{array}$ & $\begin{array}{l}\text { R.A. } \\
\text { (3) }\end{array}$ & $\begin{array}{l}\text { Decl. } \\
\text { (4) }\end{array}$ & $\begin{array}{l}i^{\prime} \\
(5)\end{array}$ & $\begin{array}{l}t(\mathrm{~s}) \\
(6)\end{array}$ & $\begin{array}{l}\text { Run } \\
\text { (7) }\end{array}$ & $\begin{array}{c}\mathrm{FWHM}_{\mathrm{H} \beta} \\
(8)\end{array}$ & $\begin{array}{c}\mathrm{FWHM}_{\mathrm{Mg} \text { II }} \\
(9)\end{array}$ & $\begin{array}{l}\sigma_{\mathrm{H} \beta} \\
(10)\end{array}$ & $\begin{array}{c}\sigma_{\mathrm{Mg} \mathrm{II}} \\
(11)\end{array}$ & $\begin{array}{l}L_{3000} \\
(12)\end{array}$ & $\begin{array}{c}L_{5100} \\
(13)\end{array}$ & $\begin{array}{l}L_{\mathrm{H} \beta} \\
(14)\end{array}$ & $\begin{array}{c}L_{\mathrm{Mg} \mathrm{II}} \\
(15)\end{array}$ \\
\hline$\overline{S 01}$ & 0.3593 & 153916.24 & +032322.07 & 18.89 & 10400 & 1,4 & $4662 \pm 26$ & $3324 \pm 66$ & $2194 \pm 21$ & $1856 \pm 35$ & $2.38 \pm 0.01$ & $1.37 \pm 0.02$ & $2.22 \pm 0.01$ & $5.25 \pm 0.05$ \\
\hline S02 & 0.3545 & 161111.66 & +513131.16 & 19.00 & 3000 & 1 & $4841 \pm 35$ & $3332 \pm 48$ & $2274 \pm 25$ & $2088 \pm 35$ & $1.61 \pm 0.01$ & $1.25 \pm 0.02$ & $2.95 \pm 0.01$ & $6.43 \pm 0.06$ \\
\hline S03 & 0.3582 & 173203.08 & +611751.89 & 18.30 & 5500 & 1,8 & $3018 \pm 17$ & $2221 \pm 42$ & $1716 \pm 9$ & $1249 \pm 26$ & $4.11 \pm 0.01$ & $2.11 \pm 0.04$ & $3.80 \pm 0.01$ & $4.14 \pm 0.04$ \\
\hline S04 & 0.3579 & 210211.50 & -064645.01 & 18.57 & 2400 & 1 & $2821 \pm 46$ & $3079 \pm 60$ & $1749 \pm 46$ & $1708 \pm 45$ & $2.05 \pm 0.03$ & $1.19 \pm 0.05$ & $1.07 \pm 0.02$ & $3.99 \pm 0.06$ \\
\hline S05 & 0.3530 & 210451.83 & -071209.41 & 18.54 & 12600 & 1,4 & $4908 \pm 26$ & $4013 \pm 114$ & $3333 \pm 17$ & $2637 \pm 90$ & $3.14 \pm 0.09$ & $2.23 \pm 0.03$ & $4.52 \pm 0.05$ & $6.58 \pm 0.14$ \\
\hline S06 & 0.3684 & 212034.18 & -064122.24 & 18.84 & 3300 & 1 & $4527 \pm 65$ & $3056 \pm 153$ & $1413 \pm 106$ & $1423 \pm 123$ & $2.44 \pm 0.02$ & $1.10 \pm 0.09$ & $1.30 \pm 0.04$ & $2.74 \pm 0.08$ \\
\hline S07 & 0.3517 & 230946.14 & +000048.87 & 18.18 & 7200 & 1,4 & $4635 \pm 28$ & $3429 \pm 125$ & $2547 \pm 15$ & $2107 \pm 55$ & $3.66 \pm 0.03$ & $1.81 \pm 0.08$ & $3.89 \pm 0.01$ & $3.50 \pm 0.05$ \\
\hline S08 & 0.3585 & 235953.44 & -093655.63 & 18.49 & 2400 & 1 & $2909 \pm 63$ & $2212 \pm 72$ & $1217 \pm 33$ & $1167 \pm 75$ & $2.89 \pm 0.02$ & $1.59 \pm 0.05$ & $0.89 \pm 0.03$ & $1.70 \pm 0.05$ \\
\hline S09 & 0.3542 & 005916.10 & +153816.10 & 18.38 & 1800 & 1 & $2655 \pm 28$ & $2946 \pm 52$ & $1748 \pm 21$ & $1652 \pm 44$ & $2.68 \pm 0.03$ & $1.76 \pm 0.06$ & $2.80 \pm 0.03$ & $6.74 \pm 0.08$ \\
\hline S10 & 0.3505 & 010112.06 & -094500.81 & 17.97 & 3300 & 1,7 & $4850 \pm 20$ & $3388 \pm 97$ & $2597 \pm 12$ & $2034 \pm 96$ & $6.65 \pm 0.06$ & $2.77 \pm 0.04$ & $6.12 \pm 0.02$ & $6.84 \pm 0.11$ \\
\hline S11 & 0.3558 & 010715.97 & -083429.37 & 18.47 & 10200 & 1,4 & $2595 \pm 14$ & $50 \pm 50$ & $1354 \pm 8$ & $1410 \pm 39$ & $3.33 \pm 0.05$ & $1.57 \pm 0.03$ & $2.55 \pm 0.01$ & $2.74 \pm 0.04$ \\
\hline $\mathrm{S} 12$ & 0.3574 & 021340.59 & +134756.05 & 18.37 & 1800 & 1 & $8800 \pm 333$ & $14 \pm 172$ & $4256 \pm 56$ & $3376 \pm 107$ & $3.58 \pm 0.05$ & $1.82 \pm 0.12$ & $4.67 \pm 0.03$ & $8.30 \pm 0.13$ \\
\hline S16 & 0.3702 & 111937.59 & +005620.36 & 19.10 & 600 & 9 & $3749 \pm 784$ & $08 \pm 704$ & $1867 \pm 445$ & $3518 \pm 579$ & $0.21 \pm 0.02$ & $0.69 \pm 0.11$ & $0.76 \pm 0.08$ & $0.41 \pm 0.03$ \\
\hline S21 & 0.3532 & 110556.18 & +031243.15 & 17.31 & 1500 & 2 & $96 \pm 145$ & $11 \pm 211$ & $3897 \pm 105$ & $2037 \pm 131$ & $1.92 \pm 0.01$ & $5.33 \pm 0.09$ & $8.10 \pm 0.03$ & $2.36 \pm 0.05$ \\
\hline S23 & 0.3511 & 140016.65 & -010822.16 & 18.16 & 1800 & 2,4 & $9629 \pm 146$ & $5482 \pm 151$ & $4251 \pm 168$ & $2604 \pm 55$ & $2.20 \pm 0.01$ & $1.78 \pm 0.03$ & $2.70 \pm 0.02$ & $4.63 \pm 0.05$ \\
\hline S24 & 0.3616 & 140034.70 & +004733.43 & 18.29 & 9600 & 2,4 & $7061 \pm 49$ & $4466 \pm 72$ & $2635 \pm 17$ & $2288 \pm 30$ & $1.81 \pm 0.03$ & $1.49 \pm 0.02$ & $2.39 \pm$ & $5.00 \pm 0.03$ \\
\hline S26 & 0.3691 & 152922.26 & +592854.54 & 18.92 & 3600 & 2 & $5386 \pm 22$ & $4642 \pm 149$ & $1914 \pm 10$ & $2305 \pm 130$ & $1.59 \pm 0.01$ & $0.83 \pm 0.02$ & $2.36 \pm 0.02$ & $2.43 \pm 0.06$ \\
\hline S27 & 0.3667 & 153651.27 & +541442.63 & 18.86 & 7200 & 2 & $2508 \pm 28$ & $2682 \pm 65$ & $1409 \pm 17$ & $1234 \pm 47$ & $1.52 \pm 0.04$ & $1.26 \pm 0.05$ & $1.72 \pm 0.01$ & $1.88 \pm 0.03$ \\
\hline S28 & 0.3679 & 161156.29 & +451610.91 & 18.63 & 5760 & 3,4 & $4600 \pm 51$ & $4974 \pm 87$ & $2532 \pm 36$ & $2690 \pm 47$ & $2.06 \pm 0.02$ & $0.97 \pm 0.03$ & $1.79 \pm 0.02$ & $4.43 \pm 0.04$ \\
\hline S29 & 0.3575 & 215841.92 & $-01 \quad 1500.32$ & 18.95 & 3600 & 3 & $3533 \pm 44$ & $3036 \pm 72$ & $1847 \pm 28$ & $1780 \pm 57$ & $1.14 \pm 0.02$ & $1.20 \pm 0.04$ & $1.48 \pm 0.01$ & $1.59 \pm 0.02$ \\
\hline S31 & 0.3505 & 101527.26 & +625911.52 & 18.15 & 9000 & 9,10 & $4012 \pm 27$ & $3099 \pm 123$ & $2117 \pm 20$ & $1887 \pm 74$ & $1.75 \pm 0.02$ & $0.93 \pm 0.03$ & $2.24 \pm 0.01$ & $1.93 \pm 0.04$ \\
\hline SS1 & 0.3566 & 080427.98 & +522306.21 & 18.55 & 9000 & 5 & $2620 \pm 49$ & $2458 \pm 71$ & $1501 \pm 32$ & $1255 \pm 66$ & $1.59 \pm 0.02$ & $1.04 \pm 0.08$ & $1.88 \pm 0.03$ & $1.62 \pm 0.03$ \\
\hline SS2 & 0.3672 & 093455.60 & +051409.15 & 18.82 & 7200 & 5 & $2815 \pm 61$ & $2777 \pm 69$ & $1316 \pm 41$ & $1296 \pm 46$ & $0.90 \pm 0.01$ & $0.83 \pm 0.04$ & $0.81 \pm 0.02$ & $0.97 \pm 0.02$ \\
\hline SS4 & 0.3630 & 095850.15 & +400342.33 & 18.74 & 5400 & 9 & $2213 \pm 35$ & $2302 \pm 29$ & $1378 \pm 15$ & $1198 \pm 21$ & $2.28 \pm 0.01$ & $1.35 \pm 0.05$ & $1.93 \pm 0.01$ & $3.83 \pm 0.03$ \\
\hline SS5 & 0.3733 & 100706.25 & +084228.41 & 18.69 & 3600 & 9 & $2790 \pm 63$ & $1954 \pm 83$ & $1612 \pm 40$ & $1092 \pm 65$ & $1.39 \pm 0.01$ & $1.40 \pm 0.04$ & $0.90 \pm 0.01$ & $0.49 \pm 0.02$ \\
\hline SS6 & 0.3584 & 102103.57 & +304755.87 & 18.92 & 5400 & 9 & $1947 \pm 21$ & $2069 \pm 86$ & $1031 \pm 13$ & $868 \pm 57$ & $1.33 \pm 0.01$ & $0.69 \pm 0.03$ & $1.12 \pm 0.01$ & $0.85 \pm 0.02$ \\
\hline SS7 & 0.3618 & 104331.50 & -010732.88 & 18.82 & 5400 & 9 & $2959 \pm 56$ & $2020 \pm 79$ & $1371 \pm 27$ & $1163 \pm 47$ & $2.06 \pm 0.01$ & $0.98 \pm 0.04$ & $1.19 \pm 0.01$ & $1.44 \pm 0.03$ \\
\hline SS8 & 0.3656 & 104610.60 & +035031.26 & 18.45 & 9900 & $9,10,11$ & $2733 \pm 43$ & $2446 \pm 48$ & $1532 \pm 11$ & $1298 \pm 28$ & $3.20 \pm 0.01$ & $1.54 \pm 0.02$ & $2.27 \pm 0.01$ & $3.03 \pm 0.03$ \\
\hline SS9 & 0.3701 & 125838.71 & +455515.55 & 18.56 & 5400 & 9 & $2787 \pm 27$ & $3014 \pm 50$ & $1569 \pm 14$ & $1501 \pm 25$ & $2.87 \pm 0.04$ & $1.25 \pm 0.02$ & $2.08 \pm 0.02$ & $4.25 \pm 0.04$ \\
\hline SS10 & 0.3658 & 133414.84 & +114221.52 & 17.83 & 3600 & 10 & $2232 \pm 36$ & $1429 \pm 58$ & $1431 \pm 20$ & $820 \pm 42$ & $4.97 \pm 0.13$ & $4.09 \pm 0.13$ & $6.19 \pm 0.02$ & $4.87 \pm 0.12$ \\
\hline SS11 & 0.3732 & 135226.90 & +392426.84 & 18.39 & 2400 & 10 & $3505 \pm 78$ & $2661 \pm 117$ & $1466 \pm 70$ & $1630 \pm 96$ & $2.67 \pm 0.02$ & $2.07 \pm 0.02$ & $1.01 \pm 0.04$ & $1.84 \pm 0.06$ \\
\hline SS12 & 0.3625 & 150116.82 & +533102.13 & 17.80 & 5500 & 7 & $2101 \pm 10$ & $1865 \pm 94$ & $1371 \pm 6$ & $930 \pm 59$ & $3.34 \pm 0.04$ & $4.34 \pm 0.02$ & $6.87 \pm 0.01$ & $1.87 \pm 0.05$ \\
\hline SS13 & 0.3745 & 150541.78 & +493519.99 & 18.73 & 11100 & 8,9 & $2169 \pm 12$ & $2182 \pm 35$ & $1143 \pm 9$ & $1123 \pm 28$ & $2.16 \pm 0.01$ & $1.49 \pm 0.02$ & $1.10 \pm 0.01$ & $1.76 \pm 0.02$ \\
\hline SS14 & 0.3706 & 211531.68 & -072627.50 & 19.24 & 9000 & 7 & $2143 \pm 27$ & $2114 \pm 50$ & $1212 \pm 17$ & $1061 \pm 36$ & $1.21 \pm 0.01$ & $0.65 \pm 0.03$ & $1.05 \pm 0.01$ & $1.46 \pm 0.02$ \\
\hline SS15 & 0.3595 & 014412.77 & -000610.54 & 19.46 & 8700 & 7 & $1604 \pm 28$ & $1870 \pm 93$ & $1000 \pm 15$ & $1008 \pm 80$ & $0.46 \pm 0.00$ & $0.64 \pm 0.02$ & $0.65 \pm 0.01$ & $0.19 \pm 0.01$ \\
\hline SS17 & 0.3554 & 214410.62 & -010113.42 & 18.47 & 5400 & 7 & $1631 \pm 82$ & $1730 \pm 34$ & $1029 \pm 51$ & $819 \pm 28$ & $3.64 \pm 0.02$ & $1.90 \pm 0.10$ & $1.01 \pm 0.01$ & $3.10 \pm 0.04$ \\
\hline SS18 & 0.3582 & 234050.52 & +010635.47 & 18.50 & 7200 & 7 & $1890 \pm 179$ & $1484 \pm 36$ & $957 \pm 50$ & $756 \pm 38$ & $2.39 \pm 0.02$ & $1.36 \pm 0.03$ & $1.91 \pm 0.02$ & $1.59 \pm 0.04$ \\
\hline W01 & 0.5736 & 083654.98 & +075712.46 & 18.59 & 10800 & 6 & $7378 \pm 43$ & $5904 \pm 53$ & $3152 \pm 9$ & $3004 \pm 24$ & $5.28 \pm 0.09$ & $4.71 \pm 0.00$ & $7.06 \pm 0.03$ & $7.52 \pm 0.04$ \\
\hline W02 & 0.5720 & 110641.86 & +614146.57 & 18.96 & 12600 & 6 & $12647 \pm 88$ & $4573 \pm 340$ & $4811 \pm 34$ & $3137 \pm 50$ & $3.75 \pm 0.01$ & $3.03 \pm 0.08$ & $6.79 \pm 0.02$ & $7.57 \pm 0.20$ \\
\hline W03 & 0.5760 & 002005.69 & -005016.25 & 19.38 & 10800 & 7 & $7461 \pm 61$ & $4400 \pm 50$ & $3508 \pm 27$ & $2299 \pm 26$ & $1.78 \pm 0.01$ & $1.47 \pm 0.04$ & $3.13 \pm 0.01$ & $3.52 \pm 0.02$ \\
\hline W04 & 0.5766 & 093210.96 & +433813.03 & 18.96 & 16200 & 5,6 & $3490 \pm 30$ & $3103 \pm 184$ & $1728 \pm 18$ & $1607 \pm 83$ & $4.34 \pm 0.01$ & $3.68 \pm 0.04$ & $5.04 \pm 0.06$ & $6.32 \pm 0.15$ \\
\hline W05 & 0.5767 & 094852.73 & +363120.55 & 18.59 & 10800 & 5 & $2722 \pm 13$ & $1840 \pm 51$ & $1738 \pm 8$ & $977 \pm 28$ & $4.66 \pm 0.01$ & $4.94 \pm 0.03$ & $8.30 \pm 0.03$ & $4.19 \pm 0.06$ \\
\hline W08 & 0.5712 & 163252.42 & +263749.11 & 18.70 & 6800 & 8 & $7340 \pm 50$ & $5582 \pm 158$ & $2977 \pm 23$ & $3390 \pm 112$ & $5.08 \pm 0.02$ & $4.17 \pm 0.06$ & $5.86 \pm 0.04$ & $7.46 \pm 0.08$ \\
\hline W09 & 0.5654 & 155227.81 & +562236.46 & 19.04 & 9200 & 6 & $5273 \pm 86$ & $2673 \pm 85$ & $2747 \pm 47$ & $1594 \pm 97$ & $1.34 \pm 0.01$ & $2.64 \pm 0.05$ & $3.79 \pm 0.03$ & $1.08 \pm 0.03$ \\
\hline W10 & 0.5711 & 111415.83 & -005920.41 & 19.60 & 7200 & 5 & $3636 \pm 83$ & $2775 \pm 184$ & $1477 \pm 58$ & $1488 \pm 203$ & $1.40 \pm 0.01$ & $2.92 \pm 0.04$ & $2.12 \pm 0.05$ & $0.65 \pm 0.04$ \\
\hline W11 & 0.5650 & 015516.18 & -094555.94 & 20.09 & 10800 & 5 & $3812 \pm 89$ & $3593 \pm 115$ & $2026 \pm 72$ & $1693 \pm 114$ & $1.76 \pm 0.03$ & $0.78 \pm 0.06$ & $1.39 \pm 0.04$ & $2.24 \pm 0.05$ \\
\hline W12 & 0.5623 & 143955.10 & +355305.31 & 19.20 & 9000 & 6 & $7698 \pm 221$ & $2370 \pm 82$ & $3859 \pm 27$ & $1769 \pm 46$ & $3.96 \pm 0.01$ & $3.62 \pm 0.09$ & $9.01 \pm 0.02$ & $7.83 \pm 0.11$ \\
\hline
\end{tabular}


Table 1

(Continued)

\begin{tabular}{|c|c|c|c|c|c|c|c|c|c|c|c|c|c|c|}
\hline $\begin{array}{l}\text { Name } \\
\text { (1) }\end{array}$ & $\begin{array}{c}z \\
(2)\end{array}$ & $\begin{array}{l}\text { R.A. } \\
\text { (3) }\end{array}$ & $\begin{array}{l}\text { Decl. } \\
(4)\end{array}$ & $\begin{array}{l}i^{\prime} \\
(5)\end{array}$ & $\begin{array}{l}t(\mathrm{~s}) \\
(6)\end{array}$ & $\begin{array}{l}\text { Run } \\
\text { (7) }\end{array}$ & $\begin{array}{c}\mathrm{FWHM}_{\mathrm{H} \beta} \\
(8)\end{array}$ & $\begin{array}{c}\mathrm{FWHM}_{\mathrm{Mg} \mathrm{II}} \\
(9)\end{array}$ & $\begin{array}{l}\sigma_{\mathrm{H} \beta} \\
(10)\end{array}$ & $\begin{array}{c}\sigma_{\mathrm{Mg} \mathrm{II}} \\
(11)\end{array}$ & $\begin{array}{l}L_{3000} \\
(12)\end{array}$ & $\begin{array}{l}L_{5100} \\
(13)\end{array}$ & $\begin{array}{l}L_{\mathrm{H} \beta} \\
(14)\end{array}$ & $\begin{array}{l}L_{\mathrm{Mg} \mathrm{II}} \\
(15)\end{array}$ \\
\hline W14 & 0.5617 & 125631.89 & -023130.60 & 18.77 & 3000 & 5 & $5001 \pm 15$ & $3042 \pm 102$ & $2616 \pm 16$ & $1747 \pm 65$ & $5.04 \pm 0.06$ & $5.56 \pm 0.03$ & $11.29 \pm 0.04$ & $4.16 \pm 0.07$ \\
\hline W16 & 0.5780 & 152654.93 & -003243.27 & 19.99 & 7500 & 8 & $2392 \pm 19$ & $2331 \pm 44$ & $1564 \pm 17$ & $1121 \pm 34$ & $2.30 \pm 0.01$ & $1.05 \pm 0.05$ & $2.16 \pm 0.01$ & $2.34 \pm 0.03$ \\
\hline W17 & 0.5617 & 100728.38 & +392651.81 & 19.75 & 12800 & 5,6 & $5556 \pm 94$ & $3807 \pm 97$ & $2483 \pm 59$ & $2153 \pm 36$ & $0.92 \pm 0.01$ & $0.86 \pm 0.03$ & $1.52 \pm 0.02$ & $3.06 \pm 0.03$ \\
\hline W20 & 0.5761 & 150014.81 & +322940.38 & 19.60 & 5400 & 7 & $10861 \pm 360$ & $3846 \pm 256$ & $3806 \pm 77$ & $2438 \pm 51$ & $1.18 \pm 0.01$ & $1.33 \pm 0.20$ & $1.28 \pm 0.03$ & $3.51 \pm 0.07$ \\
\hline W22 & 0.5652 & 034229.70 & -052319.44 & 18.70 & 9000 & 5 & $5835 \pm 80$ & $3344 \pm 54$ & $2654 \pm 20$ & $2044 \pm 35$ & $6.03 \pm 0.01$ & $4.65 \pm 0.05$ & $5.98 \pm 0.04$ & $4.89 \pm 0.04$ \\
\hline
\end{tabular}

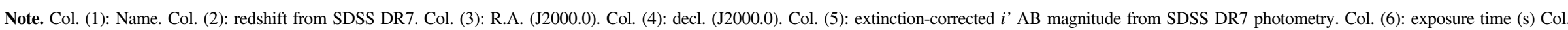

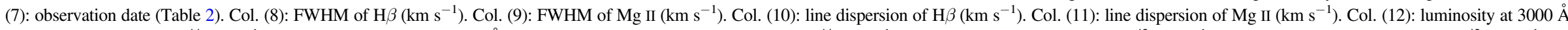

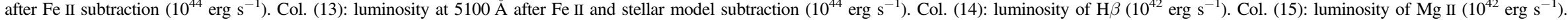


photoionization assumption (Wandel et al. 1999), while for other luminosity measures, i.e., UV continuum or line luminosities, the value can also vary.

The alternative UV mass estimators are calibrated compared to the $\mathrm{H} \beta$-based mass estimators. Since the directly measured time-lag (i.e., BLR size) based on the variability of $\mathrm{Mg}$ II has been limited to only a small number of objects (Reichert et al. 1994; Metzroth et al. 2006; Shen et al. 2016), due to the observational difficulties and/or the lack of consistent variability between line and continuum (Woo 2008; Cackett et al. 2015), there is no available Mg II-based size-luminosity relation. Thus, UV mass estimators need to be calibrated with $\mathrm{H} \beta$-based measurements, i.e., time lags and mass estimates. Using the reverberation-mapped AGN sample, for example, McLure \& Jarvis (2002) compared the H $\beta$-based BLR size with the UV luminosity at $3000 \AA\left(L_{3000}\right)$, and provided a recipe of $M_{\mathrm{BH}}$ determination based on $L_{3000}$ and the FWHM of Mg II, (see also McLure \& Dunlop 2004; Vestergaard \& Peterson 2006). Note that, while the AGNs with the measured reverberation lags and the line width measurements based on the rms spectra have been used to calibrate $M_{\mathrm{BH}}$ estimators, this sample is limited to relatively low- $z$ objects with low-tomoderate luminosity (Bentz et al. 2006). Alternatively, the calibration has been performed by determining the best coefficients in Equation (1), which provides the most consistent masses compared to $\mathrm{H} \beta$-based single-epoch masses (e.g., McGill et al. 2008; Shen et al. 2011).

In this work, we present the UV mass estimators by updating the result of McGill et al. (2008), who reported a new calibration of $\mathrm{Mg}$ II-based mass estimators using a small sample of 19 AGNs with very high-quality Keck spectroscopic data, and investigated the systematic uncertainties due to the variability, line width measurements, and Eddington ratio, which may affect the calibration of UV mass estimators. The improvements in the current work are: (i) the enlarged sample size from 19 AGNs to $\sim 50$ objects; (ii) the enlarged dynamic range by a factor of $\sim 2$; (iii) the improved and updated spectral decomposition, particularly with a better UV Fe II template; (iv) the updated virial factor and normalization, reflecting the progress of the calibration of $\mathrm{H} \beta$-based $M_{\mathrm{BH}}$ studies. The highquality spectra from the Keck telescope enable us to reliably remove non-broad line components from the observed spectra to accurately measure the continuum and emission line properties. In Section 2, we describe the sample, observation, and data reduction. Section 3 describes the fitting procedure and analysis. Section 4 presents the calibration. Finally, we provide a discussion and summary in Sections 5 and 6 , respectively. The following cosmological parameters are used throughout the paper: $H_{0}=70 \mathrm{~km} \mathrm{~s}^{-1} \mathrm{Mpc}^{-1}, \Omega_{\mathrm{m}}=0.30$, and $\Omega_{\Lambda}=0.70$.

\section{Observations and Data Reduction}

The sample was initially selected for measuring stellar velocity dispersions of AGN host galaxies to study the evolution of the $M_{\mathrm{BH}}-\sigma_{*}$ relation (Treu et al. 2004; Woo et al. 2006; Woo 2008; Bennert et al. 2010). We selected moderate-luminosity AGNs from the SDSS, at particular redshift ranges, at $\sim 0.36$ and $\sim 0.52$ in order to observe the broad $\mathrm{H} \beta$ emission line and stellar absorption lines in the rest frame 5100-5500

We observed the sample at the Keck telescope between 2003 September and 2009 April as summarized in Table 1 using the
Table 2

Observation Log

\begin{tabular}{lcccc}
\hline \hline Run & Date & $\begin{array}{c}\text { Slit } \\
(\operatorname{arcsec}) \\
(3)\end{array}$ & $\begin{array}{c}\text { Seeing } \\
(\operatorname{arcsec})\end{array}$ & Weather \\
$(1)$ & $(2)$ & 1.5 & $\sim 1$ & $(5)$ \\
\hline 1 & 2003 Sep 3 & 1 & $\sim 1$ & Cirrus \\
2 & 2004 May 14 & 1 & $\sim 0.8$ & Clear \\
3 & 2004 May 22 & 1 & $\sim 0.7-0.9$ & Clear \\
4 & 2005 Jul 7, 8 & 1 & $\sim 0.6-1.2$ & Clear \\
5 & 2007 Jan 23, 24, 25 & 1 & $\sim 0.6-0.8$ & Clear \\
6 & 2007 Apr 19, 20, 21 & 1 & $\sim 1-1.7$ & Clear \\
7 & 2007 Aug 18, 19 & 1 & $\sim 0.8$ & Clear \\
8 & 2008 Aug 2, 3 & 1 & $\sim 1.1-1.5$ & Clear \\
9 & 2009 Jan 21, 22 & 1 & $\sim 1.2$ & Cirrus \\
10 & 2009 Apr 2 & 1 & $\sim 0.8$ & Clear \\
11 & 2009 Apr 16 & & & \\
\hline
\end{tabular}

Note. Col. (1): observing run. Col. (2): observing date. Col. (3): slit width. Col. (4): seeing. Col. (5): weather condition.

Low-resolution Imaging Spectrometer (Oke et al. 1995), which provided two spectral ranges, containing $\mathrm{Mg}$ II $(2798 \AA)$ and $\mathrm{H} \beta$ (4861 $\AA$ ) broad emission lines at blue and red CCDs, respectively. The data reduction and calibration for the red and blue CCD data were reported by Woo et al. (2006) and McGill et al. (2008), respectively. Here, we briefly summarize the observations and reduction for the blue CCD (see Table 2). We used the 600 lines $\mathrm{mm}^{-1}$ grism with a pixel scale of $0.63 \mathrm{~A}$ and a resolution of $145 \mathrm{~km} \mathrm{~s}^{-1}$ in line dispersion.

Standard spectroscopic data reduction processes, including bias subtraction, flat-fielding, flux calibration, and wavelength calibration were performed using IRAF. ${ }^{5}$ We extracted onedimensional spectra with a 10 pixel (i.e., 1".35) wide aperture. Wavelength calibration was applied using $\mathrm{Hg}, \mathrm{Ne}$, and $\mathrm{Cd}$ arc lamp images. After the flux calibration based on the observation of spectroscopic standard stars (i.e., Feige 34), we rescaled the flux level of our targets to that of SDSS spectrophotometry to compensate the uncertainties of flux calibration due to slit loss, seeing effect, etc. Finally, Galactic extinction was corrected for based on the method given by Schlegel et al. (1998) (see Figure 1).

\section{Measurements}

We measured the line width of $\mathrm{H} \beta$ and $\mathrm{Mg}$ II and the luminosity of the AGN continuum at 5100 and $3000 \AA$ as well as $\mathrm{H} \beta$ and $\mathrm{Mg}$ II lines, based on the multi-component spectral analysis (see Table 1). Here, we describe the fitting process for $\mathrm{H} \beta$ and $\mathrm{Mg}$ II, respectively.

\section{1. $H \beta$}

To measure the properties of the broad $\mathrm{H} \beta$ line, we performed a multi-component decomposition analysis, following the procedure described by Woo et al. (2006) (see also Woo et al. 2010, 2013, 2015; Park et al. 2012b). All measurements were reported by Park et al. (2015) and here we briefly summarize the fitting procedure for completeness. First, we modeled the AGN continuum with a power law. The Fe II

\footnotetext{
5 IRAF is distributed by the National Optical Astronomy Observatories, which are operated by the Association of Universities for Research in Astronomy, Inc., under cooperative agreement with the National Science Foundation (NSF).
} 

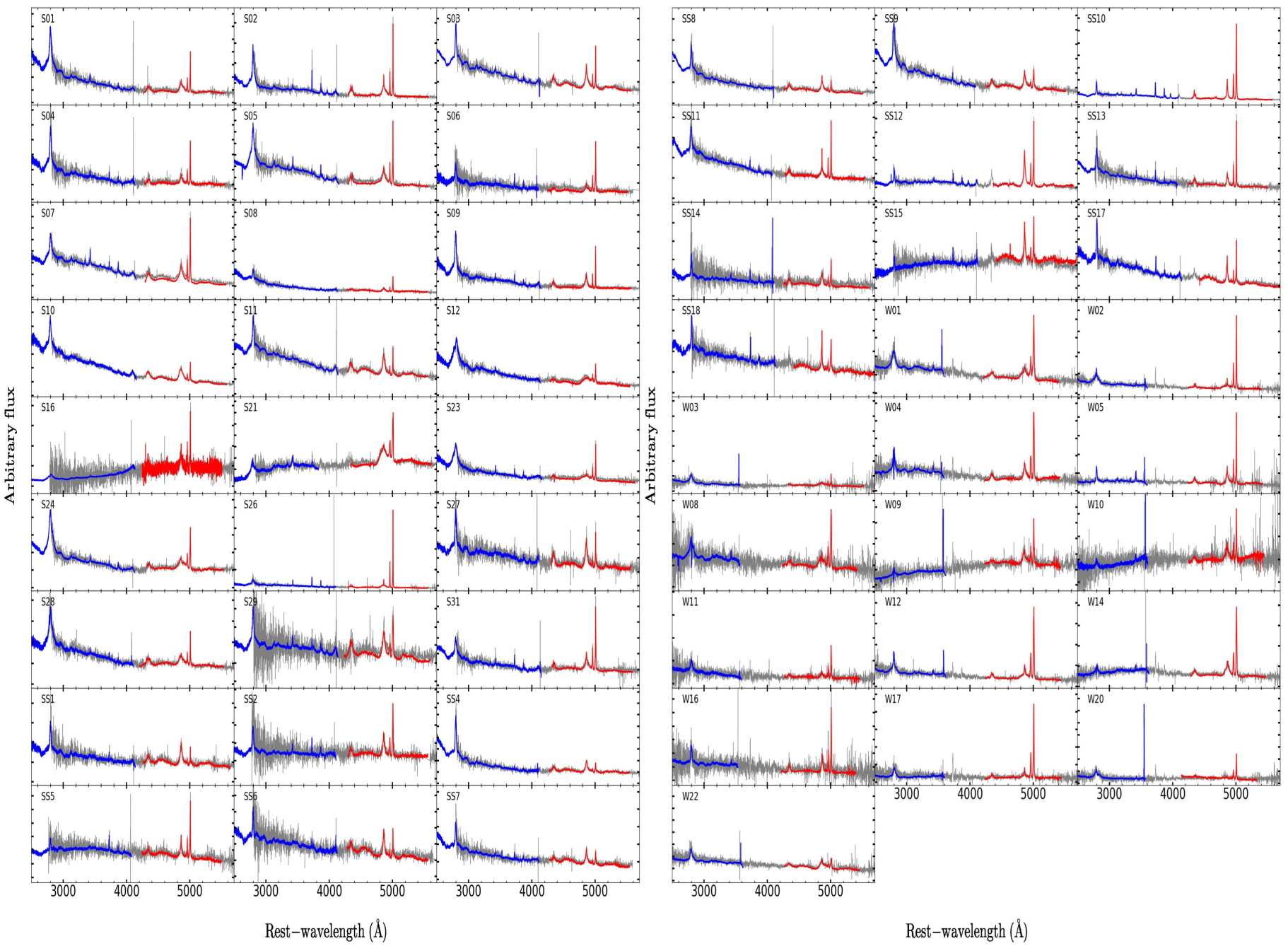

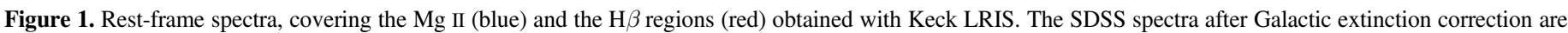
shown in gray.

models were constructed by broadening the I Zwicky 1 template from Boroson \& Green (1992) with a series of Gaussian velocities, while the stellar component was fitted with a simple stellar population synthesis model of Bruzual \& Charlot (2003) with solar metallicity and age of 11 Gyr. The stellar model improves the $\mathrm{H} \beta$ line fitting since the $\mathrm{H} \beta$ absorption line attributed from the stellar component is blended with a peak of the $\mathrm{H} \beta$ emission line originating from the AGN. In particular, the FWHM of the line profile is sensitive to the shape of the peak while the line dispersion is not significantly affected.

The fitting process was carried out using the nonlinear Levenberg-Marquardt least-squares fitting routine mpfit (Markwardt 2009) in IDL, using two spectral fitting regions: 4430-4730 $\AA$ and 5100-5400 $\mathrm{A}$, where the Fe II blends are strong. The blue end of the fitting regions were slightly adjusted to avoid $\mathrm{H} \gamma$ and $\mathrm{H} \beta$ contamination if necessary.

For the broad component of $\mathrm{H} \beta$, we used a sixth-order Gauss-Hermite series model. If the broad component of $\mathrm{H} \beta$ was blended with the He II $\lambda 4686 \AA$ line, we fitted the He II line, using two single Gaussian models, respectively for the broad and narrow components of He II, simultaneously with the $\mathrm{H} \beta$ model. We separately fitted the narrow component of $\mathrm{H} \beta$ using the best-fit model of [O III] $5007 \AA$. Based on the best model of the broad $\mathrm{H} \beta$ component, we measured FWHM, line dispersion $\left(\sigma_{\mathrm{H} \beta}\right)$, and line luminosity. We also measured the monochromatic luminosity at $5100 \AA\left(L_{5100}\right)$ by averaging the continuum flux in the $50 \AA$ window, using the power-law model representing the AGN continuum. The measurement errors of the line width and continuum luminosity were determined based on Monte Carlo simulations by generating 100 mock spectra by randomly fluctuating fluxes using the flux errors, and performing the decomposition analysis for each spectrum. We used the 1- $\sigma$ dispersion of the distribution as the measurement uncertainty.

\section{2. $M g I I$}

To measure the width and luminosity of the Mg II line, we followed the procedure outlined by McGill et al. (2008). The multi-component fitting procedure is similar to that applied to the $\mathrm{H} \beta$ region, and here we briefly describe the fitting process. First, we fitted the AGN power-law continuum and Fe II blends, using the two windows 2600-2750 $\AA$ and 2850-3090 A. Second, we fitted the Mg II line with a sixthorder Gauss-Hermite series. The purpose of the line fitting is to measure the flux-weighted width of the line profle, to represent the velocity distribution of the gas. Thus, while we do not 


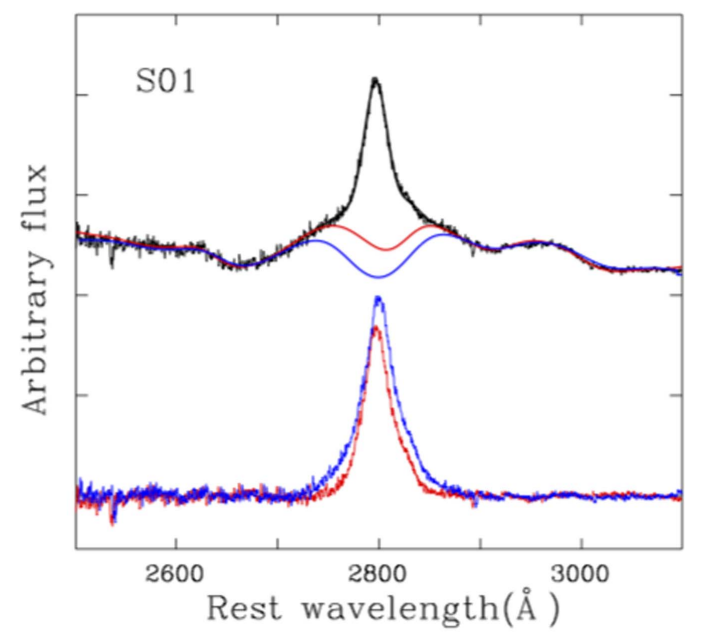

Figure 2. Top: the raw spectrum of S01 (black) along with the best-fit Fe II models, respectively, using the Fe II templates from Vestergaard \& Wilkes (2001) (blue) and Tsuzuki et al. (2006) (red). Bottom: Fe II-subtracted Mg II line profiles using the two different Fe II templates.

attempt to interpret the meaning of each coefficient in the Gauss-Hermite series, we determine the best model to reproduce the line profile. Since the Gauss-Hermite series can have negative values at the wing of the line profile, we empirically limit or adjust the fitting range, in order to prevent negative fluxes in the best-fit model.

We decided not to use a separate model to $\mathrm{ft}$ a narrow component of $\mathrm{Mg}$ II since we do not see a clear signature that suggests the presence of the narrow component in the $\mathrm{Mg}$ II line profile (see Section 5.1 for more details on narrow component subtraction). Note that the FWHM measurements of the broad $\mathrm{Mg}$ II can be underestimated if the existing narrow component in $\mathrm{Mg}$ II is not subtracted, while the effect on the line dispersion measurements will be insignificant. Several objects show strong absorption features in the $\mathrm{Mg}$ II line profile (e.g., W04, W08, W09, W14), for which we applied Gaussian models and simultaneously fitted them with the Mg II line profile. When the absorption features are close to the line center, increasing the uncertainty of the line width measurements, we checked whether the uncertainties of these objects introduce any systematic trend and found no significant effect (see Section 4.1). Third, the measurement errors of the $\mathrm{Mg}$ II line width and the continuum luminosity at $3000 \AA$ were determined based on Monte Carlo simulations. By randomly fluctuating fluxes using the flux errors, we generated 100 mock spectra and performed a decomposition analysis for each spectrum. Then the 1- $\sigma$ dispersion of the distribution was taken as the measurement uncertainty.

As investigated by Wang et al. (2009), a careful treatment is required for fitting Fe II blends in the $\mathrm{Mg}$ II region. We used the Fe II template from Vestergaard \& Wilkes (2001) in our previous study (McGill et al. 2008). However, this template contains no information on Fe II underneath the $\mathrm{Mg}$ II line because it was constructed from the observed spectrum of the narrow line Seyfert 1 galaxy, I Zwicky 1 after masking the Mg II line. Instead, Tsuzuki et al. (2006) suggested a new template based on the one-dimensional photoionization model combined with the observed spectrum of I Zw 1 . The merit of this template is that the Fe II emission at the location of $\mathrm{Mg}$ II is available. Thus, we investigated the difference of the line fitting results using the two different templates.

In Figure 2 we present an example of the best-fit results based on the Fe II templates of Vestergaard \& Wilkes (2001) (blue) and Tsuzuki et al. (2006) (red), respectively, for one AGN from our sample. There is a clear difference between the two fitting results: the best-fit $\mathrm{Mg}$ II line profile is broader and stronger when the template of Vestergaard \& Wilkes (2001) is used. This is due to the fact that there is no Fe II flux underneath the Mg II line in the Vestergaard \& Wilkes (2001) template; hence, the $\mathrm{Mg}$ II line model takes more flux from the blended region (i.e., close to the wing of $\mathrm{Mg}$ II) into the $\mathrm{Mg}$ II line flux. Thus, in the following analysis, we will use the results based on the Fe II template of Tsuzuki et al. (2006) for UV mass estimators.

Note that the template mismatch in subtracting the Fe II blends may cause additional systematic errors on the line width measurements. Wang et al. (2009) reported that the revised Fe II template of Tsuzuki et al. (2006) can provide reliable measurements within a $20 \%$ uncertainty in the case of the $\mathrm{Mg}$ II FWHM with the SDSS quality data. Thus, we expect that the uncertainty due to the template mismatch would be even smaller than $20 \%$ given the high quality of our Keck data. Considering the possibility that the errors based on the Monte Carlo simulation underestimate the true uncertainty of the line width measurements, we assume an average error of $5 \%, 10 \%$, and $20 \%$ in comparing the $\mathrm{Mg}$ II line width with that of $\mathrm{H} \beta$. We find that, regardless of the adopted errors, the best-fit slopes are consistent among each other, indicating that the fitting results are independent of the width measurement errors, unless the uncertainty is significantly larger than $20 \%$. Also, we investigate how the larger errors of the line width measurements affect the calibration of the best mass estimators. We obtain consistent results regardless of the adopted errors while the intrinsic scatter between mass estimates decreases with the increasing measurement errors, as expected (see Section 4.3.2).

The best-fit results for the $\mathrm{Mg}$ II line region based on the Fe II template by Tsuzuki et al. (2006) are presented in Figure 3, while the multicomponent fitting results for the $\mathrm{H} \beta$ region were presented by Park et al. (2015). There are several objects with relatively strong internal extinction, namely S16, S21, SS15, W09, and W10, for which the spectral slope is very different compared to that of the other AGNs in Figure 1. Thus, we will exclude these five AGNs from the $M_{\mathrm{BH}}$ estimator calibration since the luminosity and line width measurements are uncertain without a proper extinction correction. There are a couple of other AGNs with a hint of internal extinction in the $\mathrm{Mg}$ II region (e.g., SS5, SS12, W14); however, we decided to exclude only the five objects based on the spectral shape in the total UV to optical range (see further discussion in Section 4.2).

\subsection{Comparison of Line Profiles}

We directly compare the best-fit model of the $\mathrm{H} \beta$ and $\mathrm{Mg}$ II line profiles after normalizing the flux with the peak value in Figure 4. While the majority of objects show similar line profiles between $\mathrm{H} \beta$ and $\mathrm{Mg}$ II, the $\mathrm{H} \beta$ width is somewhat broader than that of $\mathrm{Mg}$ II, as previously reported. For example, Marziani et al. (2013) showed that on average $\mathrm{H} \beta$ is $20 \%$ broader than $\mathrm{Mg}$ II. However, there are cases with a much larger difference in their line profiles. In the case of S21, S24, $\mathrm{S} 23$, W02, W03, W09, W12, and W20, the $\mathrm{H} \beta$ line profile is clearly different from that of $\mathrm{Mg}$ II and the line width of $\mathrm{H} \beta$ is 


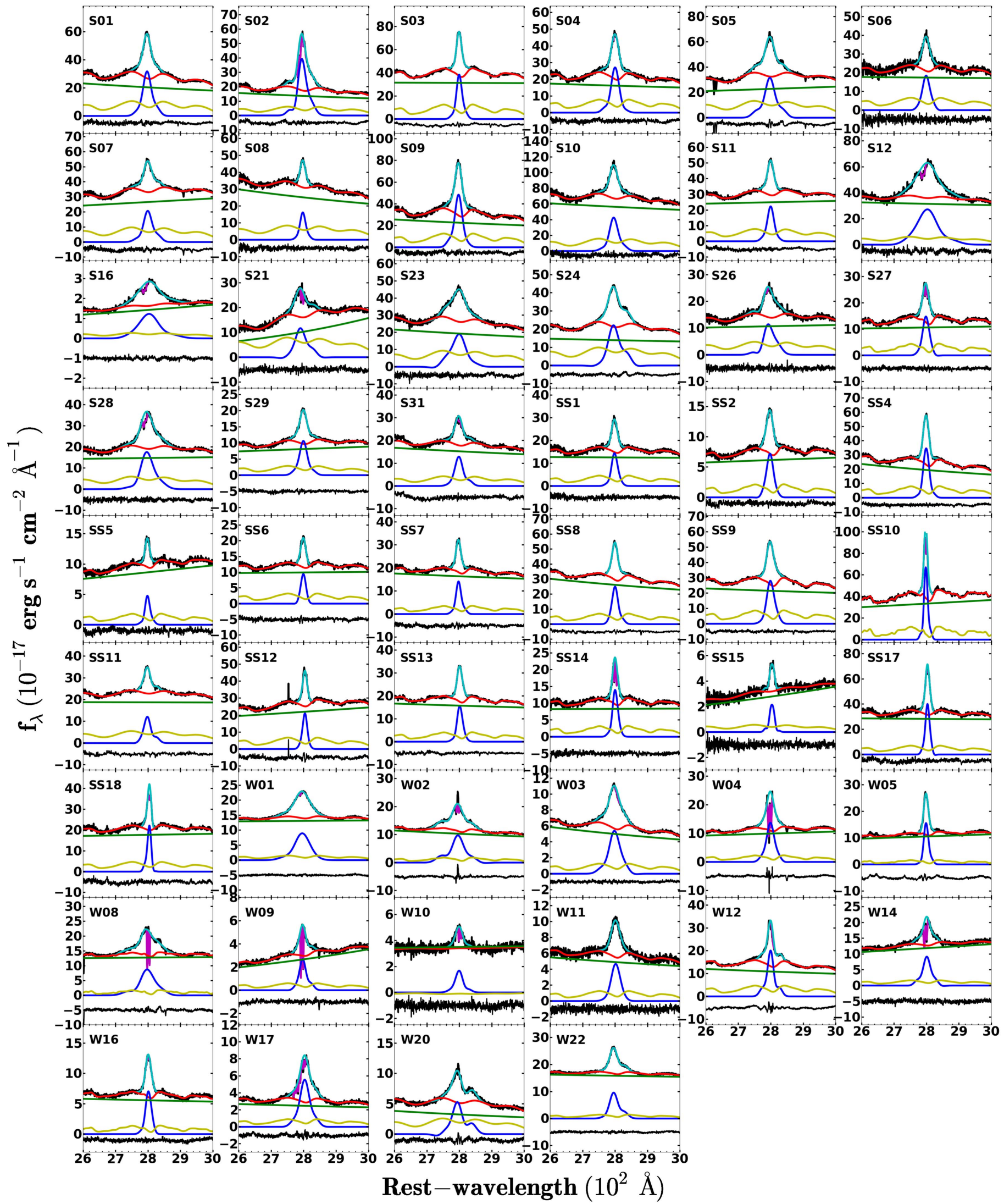

Figure 3. Multi-component fitting results for the Mg II emission line region, using the Fe II template of Tsuzuki et al. (2006). In each panel, the rest-frame Keck spectrum (thick black), power-law continuum + Fe II model (red), total model including Mg II (cyan), and models for the absorption line features in Mg II (magenta) are presented at the top, while the best-fit pseudo-continuum, i.e., AGN power-law continuum (green), Fe II model (yellow), and Mg II line model (blue) are presented in the middle. At the bottom, the residual (black) between the observed spectrum and the combined models is shown, after shifting it down arbitrarily for clarity. 


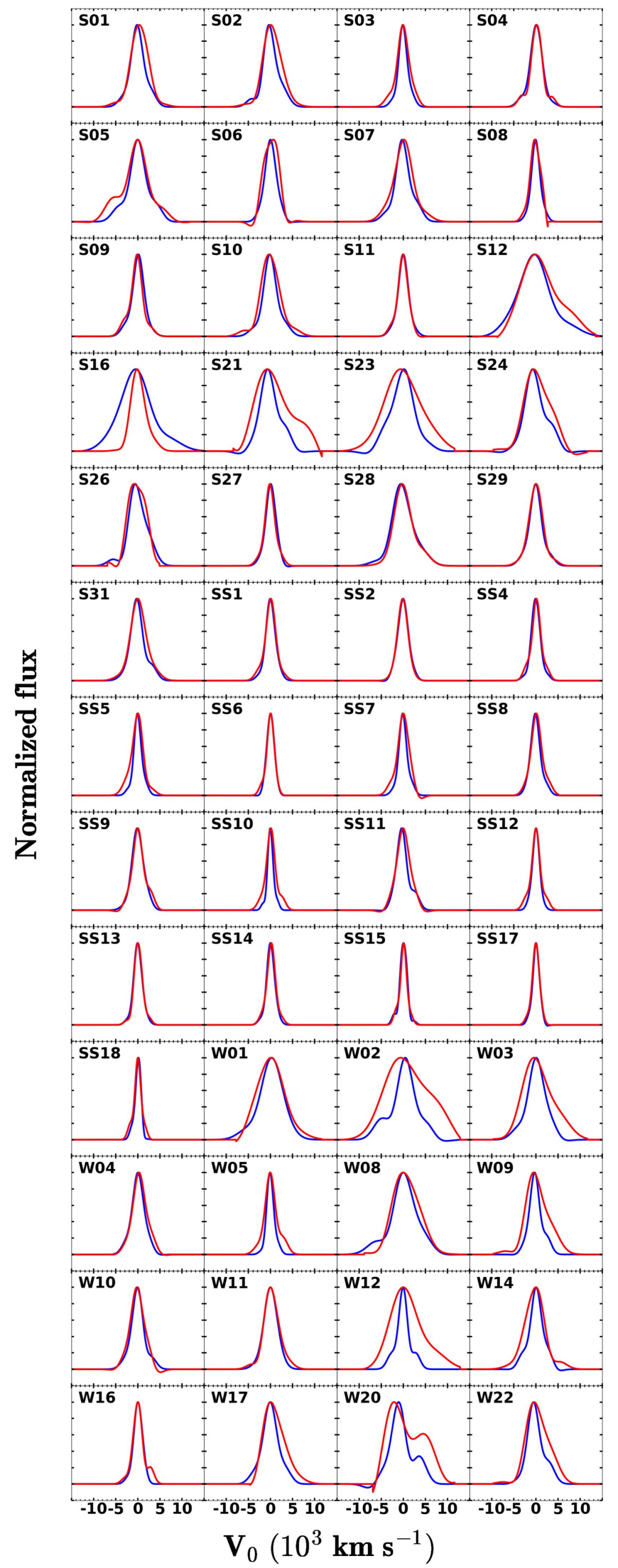

Figure 4. Comparison of the best-fit model profiles of $\mathrm{Mg}$ II (blue) and $\mathrm{H} \beta$ (red) line profiles. The flux is normalized for comparison. much broader than that of $\mathrm{Mg}$ II by more than a factor of $\sim 2$. Note that this discrepancy is mainly observed in AGNs with a very broad $\mathrm{H} \beta$ line (see the discussion on population $\mathrm{A}$ in Marziani et al. 2013). In contrast, we found that one object, $\mathrm{S} 16$, shows a much broader line in $\mathrm{Mg}$ II than in $\mathrm{H} \beta$ by a factor of $\sim 1.8$. It is not clear why the line profiles are very different between $\mathrm{H} \beta$ and $\mathrm{Mg}$ II. For the purpose of this study, we will compare the line width of $\mathrm{H} \beta$ and $\mathrm{Mg}$ II to provide UV mass estimators. However, it is clear that $M_{\mathrm{BH}}$ based on the $\mathrm{Mg}$ II line will be systematically different for these objects. If we assume that the $\mathrm{H} \beta$-based mass represents the true $M_{\mathrm{BH}}$, then the $\mathrm{Mg}$ II-based mass will suffer from systematic uncertainties due to the intrinsic difference of the line profiles between $\mathrm{H} \beta$ and $\mathrm{Mg}$ II. Thus, we will investigate the effect of these AGNs (six objects after excluding S16 and S21 due to heavy extinction) in our mass calibration (see Section 4.3).

To understand the characteristics of the line profiles, we compare line dispersion and FWHM of Mg II, using measurements based on the template of Tsuzuki et al. (2006) (Figure 5, left). The average ratio between FWHM and line dispersion $(\sigma)$ of $\mathrm{Mg}$ II is $\langle\operatorname{logFWHM} / \sigma\rangle=0.27 \pm 0.05$, corresponding to 1.86 in linear scale, which is smaller than 2.36 , the ratio of a Gaussian profile. The linear regression between FWHM and line dispersion of $\mathrm{Mg}$ II shows a slope of $0.90 \pm 0.04$, indicating that FWHM and line dispersion show an almost linear relationship. In other words, the shape of the line profile of $\mathrm{Mg}$ II does not significantly change as a function of the line width although there is a slight hint that broader $\mathrm{Mg}$ II lines tend to have broader wings and a narrower core than narrower Mg II lines.

In the case of $\mathrm{H} \beta$, the FWHM-to-line dispersion ratio is $\sim 2$ with a scatter larger than a factor of 2 . Also, the ratio increases with increasing line width, suggesting that there is a systematic difference in the line profile between AGNs with a very broad line and those with a relatively narrow line. In contrast, the $\mathrm{FWHM} / \sigma$ ratio of $\mathrm{Mg}$ II is similar to that of $\mathrm{H} \beta$, but with a much smaller scatter, indicating the $\mathrm{Mg}$ II line may not suffer a strong systematic trend as a function of line width.

\section{Calibration}

In this section we perform correlation analysis for line widths (Section 4.1), luminosities (Section 4.2), and $M_{\mathrm{BH}}$ estimates (Section 4.3), and present the best-fit results. For the regression, we use the FITEXY method as implemented by Park et al. (2012a) using MPFIT (Markwardt 2009), which finds the best-fit parameters (intercept, slope, and intrinsic scatter $\left.\sigma_{\text {int }}\right)$ by minimizing a reduced $\chi^{2}$, after accounting for measurement errors (see the detailed descriptions in Park et al. 2012a). From the Keck sample, we exclude five objects with strong internal extinction, namely S16, SS15, S21, W09, and $\mathrm{W} 10$ as we listed in Section 3.2, since the measured luminosities and line velocities are biased.

\subsection{Line Width Comparison}

We compare the width measurements of $\mathrm{Mg}$ II with those of $\mathrm{H} \beta$ in Figure 6. As the line profile of $\mathrm{Mg} \mathrm{II}$ is often different from that of $\mathrm{H} \beta$ as shown in Figure 4, we assumed an intrinsic scatter between the widths of the two lines in the fitting process. While we measured the uncertainty of the line width 

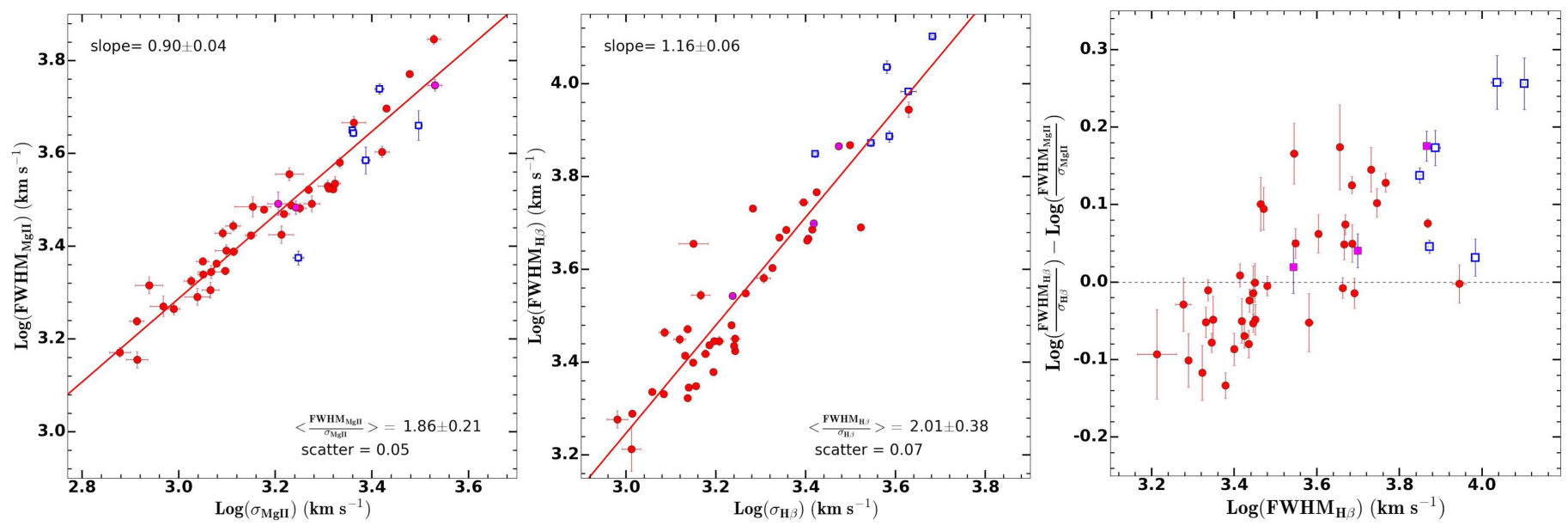

Figure 5. Comparing the FWHM and line dispersion $(\sigma)$ of $\mathrm{Mg}$ II (left) and $\mathrm{H} \beta$ (center). The objects with strong absorption line features in the Mg II line are denoted as magenta squares, showing that these objects are not deviating from the distribution of other AGNs. The six AGNs with a strong discrepancy of line profiles between $\mathrm{H} \beta$ and Mg II are shown as open blue squares, while the best fit is represented by red solid lines. Right: investigating the line profiles as a function of FWHM of Mg II and $\mathrm{H} \beta$.
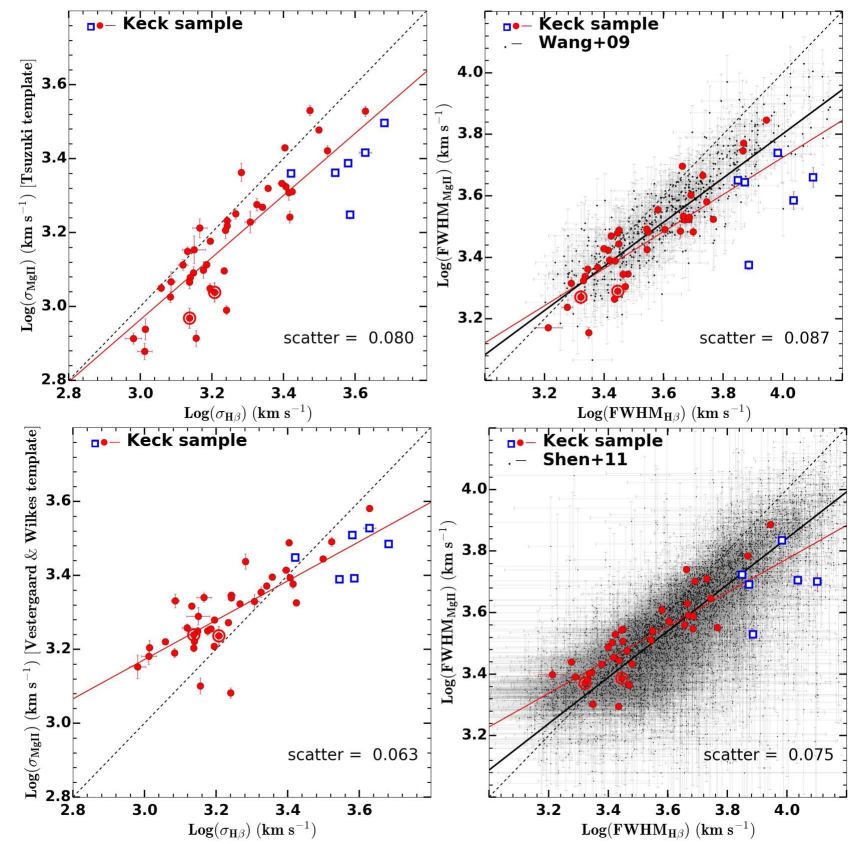

Figure 6. Comparison of the line widths of $\mathrm{H} \beta$ and $\mathrm{Mg}$ II measured based on the Tsuzuki Fe II template (top panels) and the Vestergaard \& Wilkes template (bottom panels). For FWHMs, the Keck sample (red) is plotted along with SDSS AGNs (gray), from Wang et al. (2009) (top) and Shen et al. (2011) (bottom). Note that AGNs with heavy extinction, namely S16, SS15, S21, W09, and W10 were excluded in our line regression. The solid red line represents the best-fit slope. The six AGNs with very different line profiles between $\mathrm{H} \beta$ and $\mathrm{Mg}$ II are shown as open blue squares. The AGNs with a hint of internal extinction in the Mg II region (SS5 and SS12) are marked with larger red open circles. The rms scatter of the best fit is shown as text in the plot.

measurements based on Monte Carlo simulations as discussed in Section 3.2, we also considered the systematic uncertainty and tested the fitting results assuming an average error of 5\%, $10 \%$, and $20 \%$ as the uncertainty of the width measurements of the Mg II line. We found that, regardless of the adopted errors, the best-fit slopes are consistent among each other, indicating that the fitting results are independent of the width measurement errors, unless the uncertainty is significantly large $(\gg 20 \%)$. For the final fitting results, we used the errors measured from the Monte Carlo simulations.

First, we compared $\mathrm{H} \beta$ line width and $\mathrm{Mg}$ II line width, which was measured from the spectral decomposition based on the Fe II template of Tsuzuki et al. (2006) (top panels in Figure 6). We obtained the best-fit result as

$$
\log \left(\sigma_{\mathrm{Mg} \text { II }}\right) \propto 0.84 \pm 0.07 \times \log \left(\sigma_{\mathrm{H} \beta}\right),
$$

with $\sigma_{\text {int }}=0.08 \pm 0.01$, indicating a sub-linear relationship that $\mathrm{Mg}$ II is somewhat narrower than $\mathrm{H} \beta$, particularly for AGNs with a broader line. In the case of FWHM, we obtained a shallower slope,

$$
\log \left(\mathrm{FWHM}_{\mathrm{Mg} \text { II }}\right) \propto 0.60 \pm 0.07 \times \log \left(\mathrm{FWHM}_{\mathrm{H} \beta}\right),
$$

with $\sigma_{\text {int }}=0.09 \pm 0.01$. To investigate this correlation further, we adopted a sample of 495 SDSS AGNs with $\mathrm{S} / \mathrm{N} \geqslant 20$ for $\mathrm{Mg}$ II and $\mathrm{H} \beta$ from Wang et al. (2009), who used the same Fe II template of Tsuzuki et al. (2006) in modeling the Mg II line profile, and measured the FWHM of $\mathrm{Mg}$ II and $\mathrm{H} \beta$. For the combined sample of the Keck and SDSS AGNs, we obtained the best-fit slope of $0.72 \pm 0.03\left(\sigma_{\text {int }}=0.04 \pm 0.01\right)$, which is higher than that we obtained using the Keck sample only. Since Wang et al. (2009) modeled the Mg II line profile after subtracting a narrow component of $\mathrm{Mg}$ II, it is possible that some of the FWHM measurements are systematically overestimated.

To investigate the systematic effect due to the choice of the Fe II template, we also used Mg II line width measurements based on the Fe II template of Vestergaard \& Wilkes (2001) (bottom panels in Figure 6). As described in Section 3.2, subtraction using this template introduces systematic uncertainties due to the lack of $\mathrm{Fe}$ II features at the location of the Mg II line. We found a more significant systematic difference of the line dispersions between $\mathrm{Mg}$ II and $\mathrm{H} \beta$ with the best-fit slope of $0.53 \pm 0.05\left(\sigma_{\text {int }}=0.06 \pm 0.01\right)$. These results support the hypothesis that the Fe II template of Vestergaard \& Wilkes (2001) introduces additional systematic uncertainties on the line width measurements, particularly for the line dispersion. In the case of FWHM between $\mathrm{Mg}$ II and $\mathrm{H} \beta$, we obtained the best-fit slope of $0.55 \pm 0.06\left(\sigma_{\text {int }}=0.07 \pm 0.01\right)$, which is 
similar to the slope of $0.60 \pm 0.07$ that we obtained using the Keck sample based on the Tsuzuki et al. (2006) template, indicating that the choice of Fe II template makes less significant difference in comparing the FWHM of Mg II and $\mathrm{H} \beta$. We also adopted the FWHM measurements from Shen et al. (2011), who used the Fe II template of Vestergaard \& Wilkes (2001) for the Mg II line fitting process for a large sample of type 1 AGNs, by selecting 6017 AGNs at $0.4 \leqslant z \leqslant 0.8$ with $\mathrm{S} / \mathrm{N} \geqslant 10$ in the continuum (4750 $-4950 \AA$ ). Using the combined sample of the Keck and SDSS AGNs, we obtained the best-fit slope as $0.75 \pm 0.01$ ( $\left.\sigma_{\text {int }}=0.08 \pm 0.01\right)$, which is again close to the value we obtained using the measurements of Mg II FWHM from Wang et al. (2009) based on the template of Tsuzuki et al. (2006). These results suggest that the choice of Fe II temple does not strongly affect the FWHM comparison, while it strongly changes the correlation between the line dispersions of $\mathrm{Mg}$ II and $\mathrm{H} \beta$, as expected from the fact that the two templates make a significant difference in the wing of the $\mathrm{Mg}$ II line profile.

\subsection{Luminosity Comparison}

We compare various continuum and line luminosities with the best calibrated continuum luminosity at $5100 \AA\left(L_{5100}\right)$, in order to use the UV luminosities as a proxy for BLR size. Note that including or excluding the six AGNs with very different line profiles between $\mathrm{Mg}$ II and $\mathrm{H} \beta$ does not significantly change the result. Thus, we only present the best-fit result including these AGNs. First, we compare $L_{5100}$ with $L_{\mathrm{H} \beta}$ for the Keck sample, obtaining the best-fit slope of $1.03 \pm 0.08$ with $\sigma_{\text {int }}=0.18 \pm 0.02$. This result is consistent with, but slightly shallower than, the slope of $1.13 \pm 0.01$ reported by Greene \& Ho (2005). In order to increase the dynamic range, we adopted the measurements of 6017 SDSS AGNs from Shen et al. (2011), which were used for the line width comparison in Section 4.1. For this comparison, we made an arbitrary luminosity cut at $\log \left(L_{\mathrm{H} \beta}\right)=42.7$ and $\log \left(L_{5100}\right)=44.5$ for further selecting 4584 luminous AGNs, in order to avoid uncertain $L_{5100}$ measurements of lower-luminosity AGNs since the potential contribution from stellar continuum can be significant. In fact, a systematic offset at low luminosity is clearly present in Figure 7 (gray points). By combining our Keck sample and the higher-luminosity SDSS AGNs, we obtained the best-fit result,

$$
\log \left(L_{\mathrm{H} \beta}\right) \propto 0.99 \pm 0.01 \times \log \left(L_{5100}\right)
$$

with $\sigma_{\text {int }}=0.15 \pm 0.01$, again consistent with the linear relationship.

Second, by comparing $L_{3000}$ with $L_{5100}$, we obtained the best-fit slope of $0.74 \pm 0.06\left(\sigma_{\text {int }}=0.12 \pm 0.01\right)$ for the Keck sample. To increase the dynamic range, we also adopted 4800 luminous AGNs from Shen et al. (2011), using the luminosity cut at $\log \left(L_{3000}\right)=42.62$ and $\log \left(L_{5100}\right)=45.5$, to avoid the systematic uncertainty due to stellar contribution to $L_{5100}$. In this case we obtained the best-fit result,

$$
\log \left(L_{3000}\right) \propto 1.06 \pm 0.01 \times \log \left(L_{5100}\right),
$$

with $\sigma_{\text {int }}=0.10 \pm 0.01$.

Third, by comparing the $L_{\mathrm{Mg} \text { II }}$ with $L_{5100}$, we obtained the best-fit slope of $0.62 \pm 0.13\left(\sigma_{\text {int }}=0.24 \pm 0.03\right)$ based on the Keck sample (bottom left in Figure 7). If we combined the Keck sample with the SDSS AGNs from Wang et al. (2009), who used the same Fe II template for the Mg II line fitting, we obtained the best-fit result,

$$
\log \left(L_{\mathrm{Mg} \text { II }}\right) \propto 0.87 \pm 0.03 \times \log \left(L_{5100}\right),
$$

with $\sigma_{\text {int }}=0.20 \pm 0.01$. The sub-linear correlation between Mg II and UV continuum luminosities represents the Baldwin effect (Baldwin 1977), which can be explained as due to the increase of the thermal component in the UV continuum, which is represented by the Big Blue Bump (BBB), for more luminous AGNs (Malkan \& Sargent 1982; Zheng \& Malkan 1993). Thus, for given photoionizing flux and the emission line luminosity, the continuum luminosity close to the BBB will be higher for more luminous AGNs. The sub-linear relation between Mg II and UV continuum luminosity is also reported to be related to physical parameters, i.e., Eddington ratio (see, for example, Dong et al. 2009). The linear relation between $\mathrm{H} \beta$ and optical continuum luminosity at $5100 \AA$ indicates that the effect of the increasing thermal component is relatively weak at $5100 \AA$, which is well off the BBB.

Last, we also compared $L_{\mathrm{Mg} \text { II }}$ with $L_{\mathrm{H} \beta}$ and obtained the best-fit slope of $0.66 \pm 0.11\left(\sigma_{\text {int }}=0.20 \pm 0.02\right)$ for the Keck sample. For the combined sample of Keck an SDSS AGNs from Wang et al. (2009), we obtained the best-fit slope of $0.83 \pm 0.02\left(\sigma_{\text {int }}=0.15 \pm 0.01\right)$, which is close to the slope of $L_{\mathrm{Mg} \text { II }}$ versus $L_{5100}$.

In summary, we obtained an almost linear relation between $L_{5100}$ and $L_{3000}$ while the relation is sub-linear between $L_{5100}$ and $L_{\mathrm{Mg} \text { II }}$. Also, we found that the slope between UV and optical luminosities varies depending on the sample. For $M_{\mathrm{BH}}$ calibrations, we will use the correlation results expressed using Equations (4)-(6) in the next section. However, since the correlation slope depends on the sample and the dynamic range, we will also calibrate UV mass estimators without using these correlations.

\subsection{Calibrating $M_{B H}$ Estimators}

To calibrate $M_{\mathrm{BH}}$ estimators we determine the coefficients in Equation (1) for each pair of velocity and luminosity measurements based on $\mathrm{H} \beta, \mathrm{Mg}$ II, $\mathrm{UV}$, and optical continuum, by comparing the UV-based mass with the reference $M_{\mathrm{BH}}$. As the reference mass, we adopt the $M_{\mathrm{BH}}$ estimated based on $\mathrm{H} \beta$ line dispersion and $L_{5100}$, by combining the virial theorem (i.e., $\beta=2$ ) and the $\mathrm{H} \beta$ size-luminosity relation from Bentz et al. (2013) (i.e., $\gamma=0.533)$. For $\alpha$, we adopt the virial factor $\mathrm{f}=4.47$ from Woo et al. (2015) based on the calibration of AGN $M_{\mathrm{BH}}$-stellar velocity dispersion relation, which corresponds to $\alpha=7.47$ (see the Appendix in Woo et al. 2015).

\subsubsection{H $\beta$-based Mass Estimators}

In Figure 8, we first calibrate optical mass estimators based on $\mathrm{H} \beta$. For $\mathrm{H} \beta$ line dispersion, we fixed $\beta$ as 2 (top panels), while for $\mathrm{H} \beta$ FWHM we used $\beta=2 / 1.16=1.72$ since $\log \mathrm{FWHM}_{\mathrm{H} \beta} \propto 1.16 \log \sigma_{\mathrm{H} \beta}$ (see Figure 5). Also, when we used $L_{\mathrm{H} \beta}$, we adopted the correlation result between $L_{\mathrm{H} \beta}$ and $L_{5100}$ from Equation (4), which corresponds to $\gamma=0.533$ / $0.99=0.54$. The rms scatter between two mass estimates is $\sim 0.1-0.14$ dex, indicating that the choice of velocity measure (either FWHM or line dispersion) or the choice of luminosity (i.e., either continuum luminosity at $5100 \AA$ or line luminosity of $\mathrm{H} \beta$ ) adds small additional systematic uncertainties. However, this assessment only applies when the data quality, 

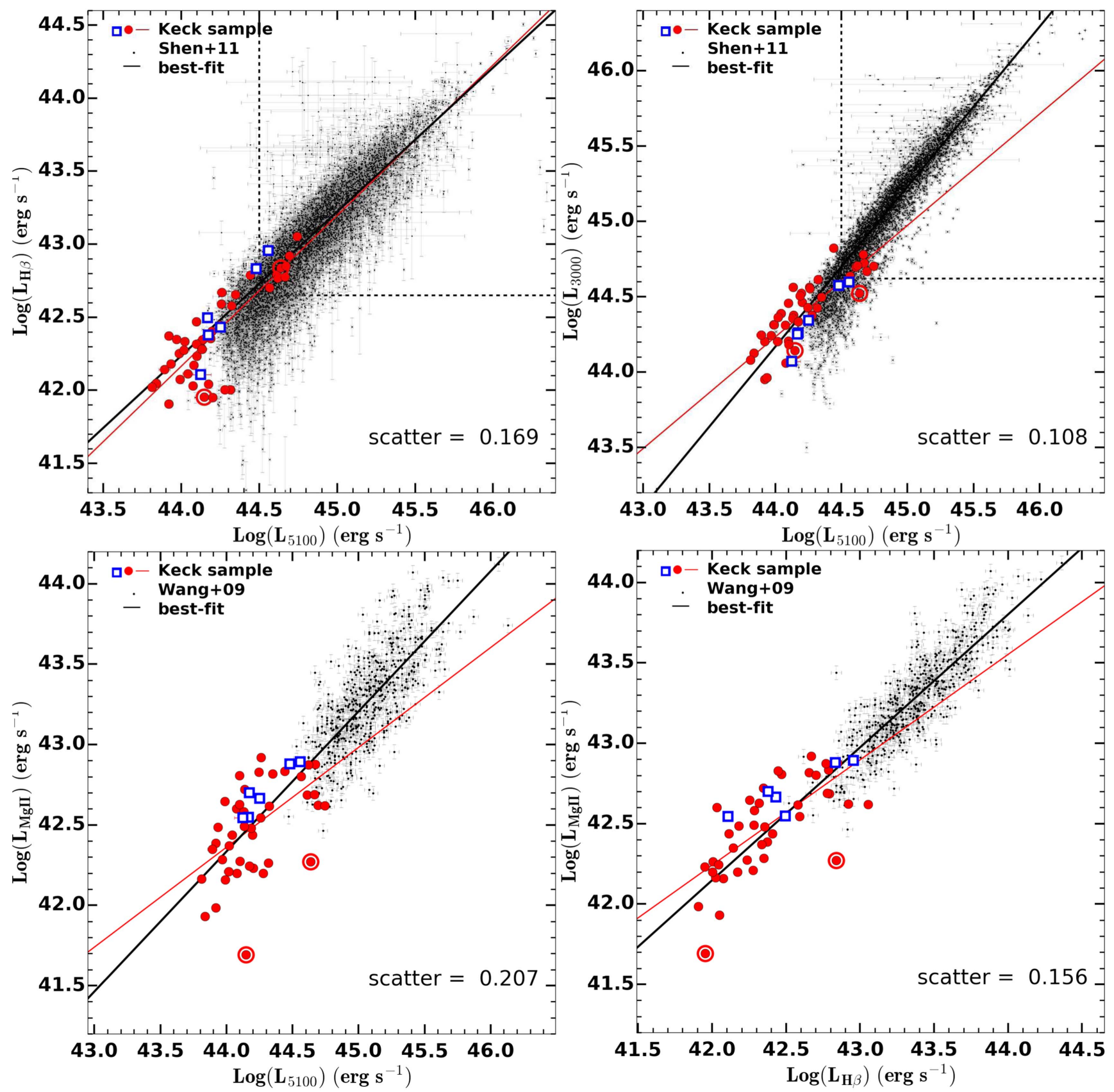

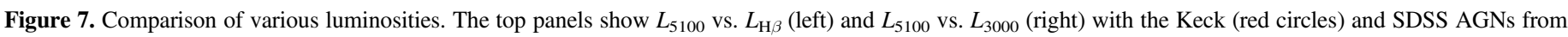

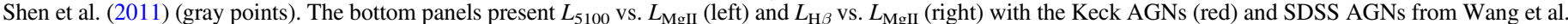

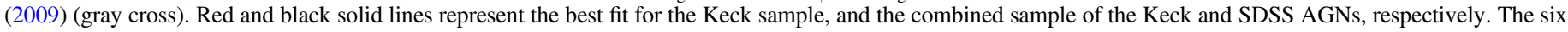

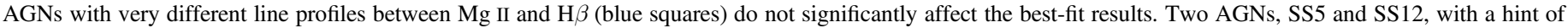
internal extinction in the $\mathrm{Mg}$ II region are marked with larger red open circles.

and hence the measurement uncertainty, is comparable to those of our Keck sample. In comparing our result based on the enlarged sample over a large dynamic range with our previous result based on the limited subsample (see Table 3 in McGill et al. 2008), we find that our new calibration is more reliable since the scatter is significantly reduced by $0.1-0.19$ dex.

\subsubsection{Mg II-based Mass Estimators}

We calibrate UV mass estimators by comparing $M_{\mathrm{BH}}$ estimated based on the $\mathrm{Mg}$ II line with the reference $M_{\mathrm{BH}}$ based on $\mathrm{H} \beta$. First, we adopt the $\beta$ value from the direct comparison of $\mathrm{H} \beta$ line dispersion with $\mathrm{Mg}$ II line dispersion as well as the $\mathrm{Mg}$ II FWHM. In other words, for $\mathrm{Mg}$ II line dispersion, $\beta=2 / 0.84=2.38$ since $\log \sigma_{\mathrm{Mg} \text { II }} \propto 0.84 \log \sigma_{\mathrm{H} \beta}$, while $\beta=2 / 0.70=2.85$ for the $\mathrm{Mg}$ II FWHM since $\log$ $\mathrm{FWHM}_{\mathrm{Mg} \text { II }} \propto 0.70 \log \sigma_{\mathrm{H} \beta}$ (see Section 4.1). For luminosity, we also use the results from Section 4.2. Since $L_{3000} \propto 1.06$ $L_{5100}$, we adopt $\gamma=0.53 / 1.06=0.50$ for $L_{3000}$. In the case of $L_{\mathrm{Mg} \text { II }}$, we use $\gamma=0.53 / 0.87=0.61$ since $L_{\mathrm{Mg} \mathrm{II}} \propto 0.87 L_{5100}$. Using these fixed $\beta$ and $\gamma$ values, we perform the $\chi^{2}$ minimization with the FITEXY method (Park et al. 2012a) to determine $\alpha$ (see Figure 9). In general the scatter is larger than 0.2 dex and the consistency with $\mathrm{H} \beta$-based mass is better for Mg II line dispersion than FWHM. Also, continuum luminosity 
Table 3

$M_{\mathrm{BH}}$ Estimators Based on $\mathrm{Mg}$ II, Using the Fiducial Mass from $\sigma_{\mathrm{H} \beta}$ and $L_{51000}$

\begin{tabular}{|c|c|c|c|c|c|c|c|c|c|c|c|}
\hline $\begin{array}{l}\text { Case } \\
\text { (1) }\end{array}$ & $\begin{array}{l}N \\
(2)\end{array}$ & $\begin{array}{c}\alpha \\
(3)\end{array}$ & $\begin{array}{c}\beta \\
(4)\end{array}$ & $\begin{array}{c}\gamma \\
(5)\end{array}$ & $\begin{array}{l}\sigma_{\text {int }} \\
(6)\end{array}$ & $\begin{array}{l}\mathrm{rms} \\
(7)\end{array}$ & $\begin{array}{c}\alpha \\
(8)\end{array}$ & $\begin{array}{c}\beta \\
(9)\end{array}$ & $\begin{array}{c}\gamma \\
(10)\end{array}$ & $\begin{array}{l}\sigma_{\text {inst }} \\
(11)\end{array}$ & $\begin{array}{l}\mathrm{rms} \\
(12)\end{array}$ \\
\hline \multicolumn{7}{|c|}{$\boldsymbol{L}_{3000}$ and $\sigma_{\mathrm{Mg} \text { II }}$} & \multicolumn{5}{|c|}{$\boldsymbol{L}_{3000}$ and $\mathbf{F W H M} \mathbf{M g I I}_{\mathrm{M}}$} \\
\hline \multirow{2}{*}{$\begin{array}{l}\text { (1) } \beta \text { and } \gamma \text { from } \\
\text { scaling }\end{array}$} & 47 & $7.48 \pm 0.03$ & $2.38 \pm 0.20$ & $0.50 \pm 0.00$ & $0.19 \pm 0.03$ & 0.23 & $6.62 \pm 0.05$ & $2.85 \pm 0.37$ & $0.50 \pm 0.00$ & $0.28 \pm 0.04$ & 0.31 \\
\hline & 41 & $7.52 \pm 0.03$ & $1.98 \pm 0.12$ & $0.50 \pm 0.00$ & $0.13 \pm 0.04$ & 0.18 & $6.83 \pm 0.03$ & $2.32 \pm 0.23$ & $0.50 \pm 0.00$ & $0.20 \pm 0.03$ & 0.23 \\
\hline $\begin{array}{l}\text { (2) } \beta=\mathbf{2} \\
\text { and } \gamma=\mathbf{0 . 5}\end{array}$ & 47 & $7.56 \pm \mathbf{0 . 0 3}$ & 2.00 & 0.50 & $\mathbf{0 . 1 7} \pm \mathbf{0 . 0 3}$ & 0.21 & $7.02 \pm 0.04$ & 2.00 & 0.50 & $\mathbf{0 . 2 3} \pm \mathbf{0 . 0 4}$ & 0.26 \\
\hline (3) $\beta=2$ & 47 & $7.48 \pm 0.06$ & 2.00 & $0.69 \pm 0.14$ & $0.17 \pm 0.03$ & 0.21 & $6.89 \pm 0.07$ & 2.00 & $0.83 \pm 0.18$ & $0.22 \pm 0.04$ & 0.25 \\
\hline (4) $\gamma=0.5$ & 47 & $7.57 \pm 0.05$ & $1.92 \pm 0.19$ & 0.50 & $0.17 \pm 0.03$ & 0.21 & $7.10 \pm 0.13$ & $1.83 \pm 0.25$ & 0.50 & $0.23 \pm 0.04$ & 0.26 \\
\hline Free $\beta$ and $\gamma$ & 47 & $7.50 \pm 0.06$ & $1.89 \pm 0.20$ & $0.70 \pm 0.15$ & $0.17 \pm 0.03$ & 0.21 & $6.97 \pm 0.12$ & $1.82 \pm 0.25$ & $0.83 \pm 0.18$ & $0.23 \pm 0.04$ & 0.25 \\
\hline \multicolumn{7}{|c|}{$\boldsymbol{L}_{\mathrm{Mg} \mathrm{II}}$ and $\sigma_{\mathrm{Mg} \mathrm{II}}$} & \multicolumn{5}{|c|}{$\boldsymbol{L}_{\mathrm{Mg} \mathrm{II}}$ and $\mathbf{F W H} \mathbf{M}_{\mathrm{MgII}}$} \\
\hline \multirow{2}{*}{$\begin{array}{l}\text { (1) } \beta \text { and } \gamma \text { from } \\
\text { scaling }\end{array}$} & 47 & $7.37 \pm 0.04$ & $2.38 \pm 0.20$ & $0.61 \pm 0.02$ & $0.25 \pm 0.03$ & 0.29 & $6.51 \pm 0.05$ & $2.85 \pm 0.37$ & $0.61 \pm 0.02$ & $0.33 \pm 0.04$ & 0.36 \\
\hline & 41 & $7.43 \pm 0.04$ & $1.98 \pm 0.12$ & $0.61 \pm 0.02$ & $0.21 \pm 0.04$ & 0.25 & $6.74 \pm 0.04$ & $2.32 \pm 0.23$ & $0.61 \pm 0.02$ & $0.26 \pm 0.04$ & 0.29 \\
\hline $\begin{array}{l}\text { (2) } \beta=\mathbf{2} \\
\text { and } \gamma=\mathbf{0 . 5}\end{array}$ & 47 & $7.50 \pm \mathbf{0 . 0 4}$ & 2.00 & 0.50 & $\mathbf{0 . 2 1} \pm \mathbf{0 . 0 3}$ & 0.24 & $6.97 \pm 0.04$ & 2.00 & 0.50 & $0.25 \pm \mathbf{0 . 0 3}$ & 0.28 \\
\hline (3) $\beta=2$ & 47 & $7.57 \pm 0.07$ & 2.00 & $0.36 \pm 0.13$ & $0.21 \pm 0.03$ & 0.24 & $6.97 \pm 0.09$ & 2.00 & $0.50 \pm 0.17$ & $0.25 \pm 0.03$ & 0.28 \\
\hline (4) $\gamma=0.5$ & 47 & $7.61 \pm 0.05$ & $1.48 \pm 0.19$ & 0.50 & $0.19 \pm 0.03$ & 0.22 & $7.27 \pm 0.13$ & $1.37 \pm 0.24$ & 0.50 & $0.23 \pm 0.03$ & 0.26 \\
\hline (5) Free $\beta$ and $\gamma$ & 47 & $7.59 \pm 0.07$ & $1.38 \pm 0.28$ & $0.59 \pm 0.20$ & $0.19 \pm 0.03$ & 0.22 & $7.26 \pm 0.12$ & $1.10 \pm 0.30$ & $0.77 \pm 0.21$ & $0.22 \pm 0.03$ & 0.25 \\
\hline
\end{tabular}

Note. Col. (1): method of calibration. Col. (2): number of data used in the calibration. Col. (3) and (8): $\alpha$ values. Col. (4) and (9): $\beta$ values. Col. (5) and (10): $\gamma$ values. Col. (6) and (11): intrinsic scatter. Col. (7) and (12): rms scatter. The recommended estimator is represented by bolded values.

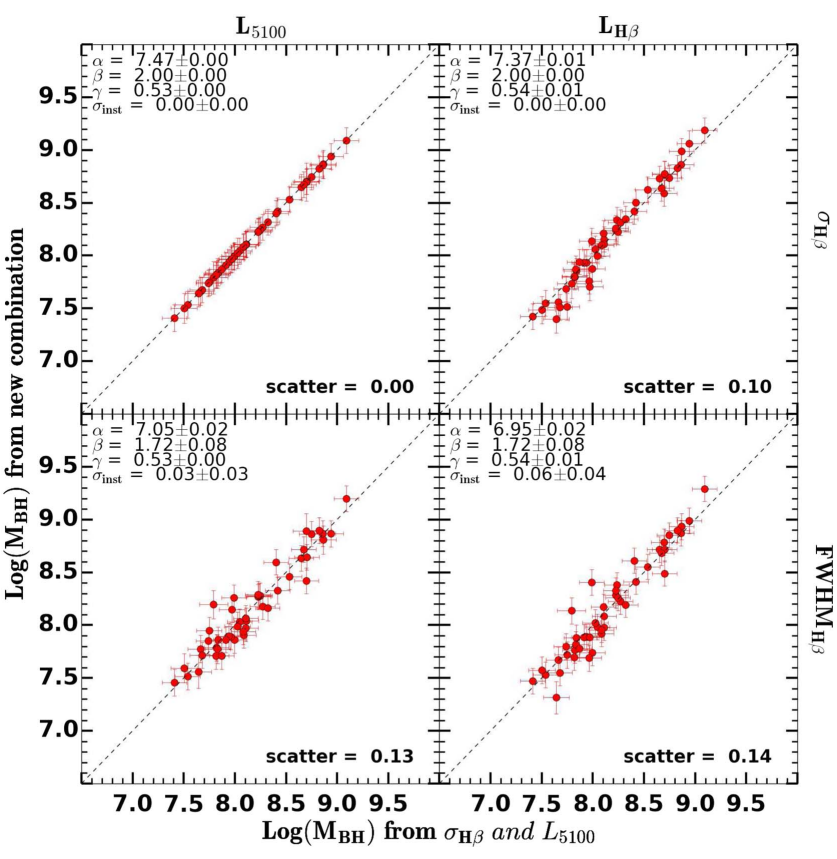

Figure 8. Cross-calibration fitting between newly derived $M_{\mathrm{BH}}$ and fiducial mass with estimators from the $\mathrm{H} \beta$ line; $x$-axis data points represent our target's fiducial mass, while $y$-axis data points are $M_{\mathrm{BH}}$ from $\alpha+\beta \log V_{1000}+\gamma \log L . V_{1000}$ means a velocity estimator using $1000 \mathrm{~km} \mathrm{~s}^{-1}$ unit, $L$ is a luminosity estimator having units $10^{44} \mathrm{erg} \mathrm{s}^{-1}$ for a continuum or $10^{42} \mathrm{erg} \mathrm{s}^{-1}$ for an emission line. $\beta$ and $\gamma$ in each panel depend on the kinds of estimators which are shown in the upper left part of the figure, and $\alpha$ is estimated by $\chi^{2}$ minimization fitting.

at $3000 \AA$ provides a better calibration than the line luminosity of $\mathrm{Mg}$ II.

Since the six AGNs with strong discrepancy of line profiles between $\mathrm{H} \beta$ and $\mathrm{Mg}$ II are more scattered from the best-fit relation as explained (open blue squares in Figure 9), we investigate how the calibration improves if we exclude them.
By removing the six AGNs, we obtained slightly better calibration with smaller scatter as presented in Table 3. Note that, after removing these six AGNs, $\beta$ becomes close to 2 as expected from a virial relation.

Second, instead of using the correlation analysis between UV and optical luminosities and line widths, we simply fix $\beta=2$ and $\gamma=0.5$, following a virial relation and the expected size-luminosity relation, regardless of the choice of velocity measure (either Mg II line dispersion or FWHM) and luminosity measure (either $L_{3000}$ or $L_{\mathrm{Mg} \text { II }}$ ) (middle panels in Figure 9). In this case, we obtained somewhat smaller scatter. For fixed $\beta$ and $\gamma$, the result does not change significantly with/ without removing the six AGNs with very different line profiles between $\mathrm{H} \beta$ and $\mathrm{Mg}$ II.

Third, we also tried to calibrate the UV mass estimators by fixing $\beta=2$ or by fixing $\gamma=0.5$. For these cases, the scatter is similar to the case with fixed $\beta$ and $\gamma$ while the coefficient $\alpha$ varies by $0.1-0.2$ dex. Finally, we let all coefficients, $\alpha, \beta$, and $\gamma$ freely vary, and obtain the best-fit results (right panel in Figure 9). Again, we do not find a significant improvement in scatter.

Based on these results we find that the pair of $\mathrm{Mg}$ II line dispersion and $L_{3000}$ provides the best calibration with a $\sim 0.2$ dex scatter than any other pair of velocity and luminosity measures. Among the various choices of $\beta$ and $\gamma$, we find no significant difference or improvement, indicating that a simple approach assuming a virial relation (i.e., $\beta=2$ ) and the expected size-luminosity relation (i.e., $\gamma=0.5$ ) is comparable to the calibration based on UV-optical comparisons of luminosities and velocities, or to the calibration using the $\alpha$, $\beta$, and $\gamma$ coefficients as free parameters.

Compared to our previous results based on a sub-sample of the current data (McGill et al. 2008), we obtain improved calibrations with smaller intrinsic scatters. The intrinsic scatter between $\mathrm{H} \beta$-based and $\mathrm{Mg}$ II-based masses is around $0.17-0.28$ dex while the rms scatter is around $0.2-0.3$ dex. For the best 

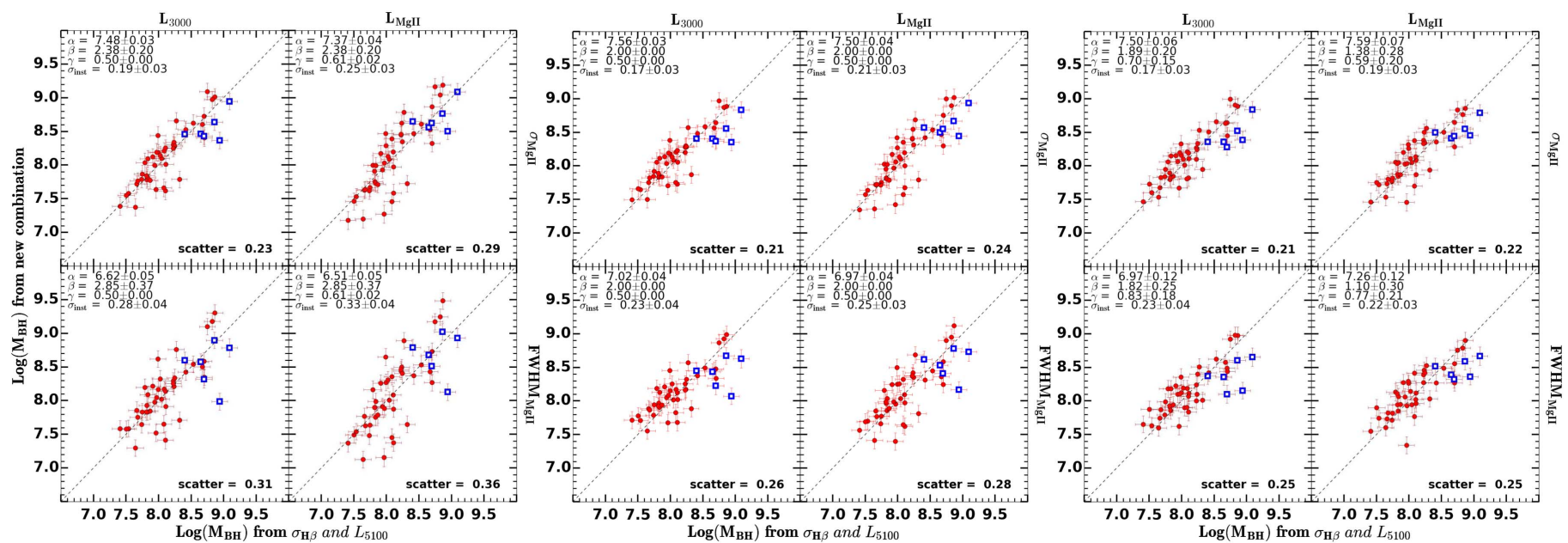

Figure 9. Similar to Figure 8 but with estimators from the Mg II line. Left panels: $\beta$ and $\gamma$ obtained from the results of line width and luminosity comparison in Sections 4.1 and 4.2. Middle panels: we fix $\beta=2$ and $\gamma=0.5$, following a virial relation and the expected size-luminosity relation. Right panels: $\beta$ and $\gamma$ from the best-fit results. Open blue squares show the six AGNs with very different line profiles between $\mathrm{H} \beta$ and $\mathrm{Mg}$ II.

Table 4

$M_{\mathrm{BH}}$ Estimators Based on $\mathrm{Mg}$ II, Using the Fiducial Mass from $\mathrm{FWHM}_{\mathrm{H} \beta}$ and $L_{51000}$

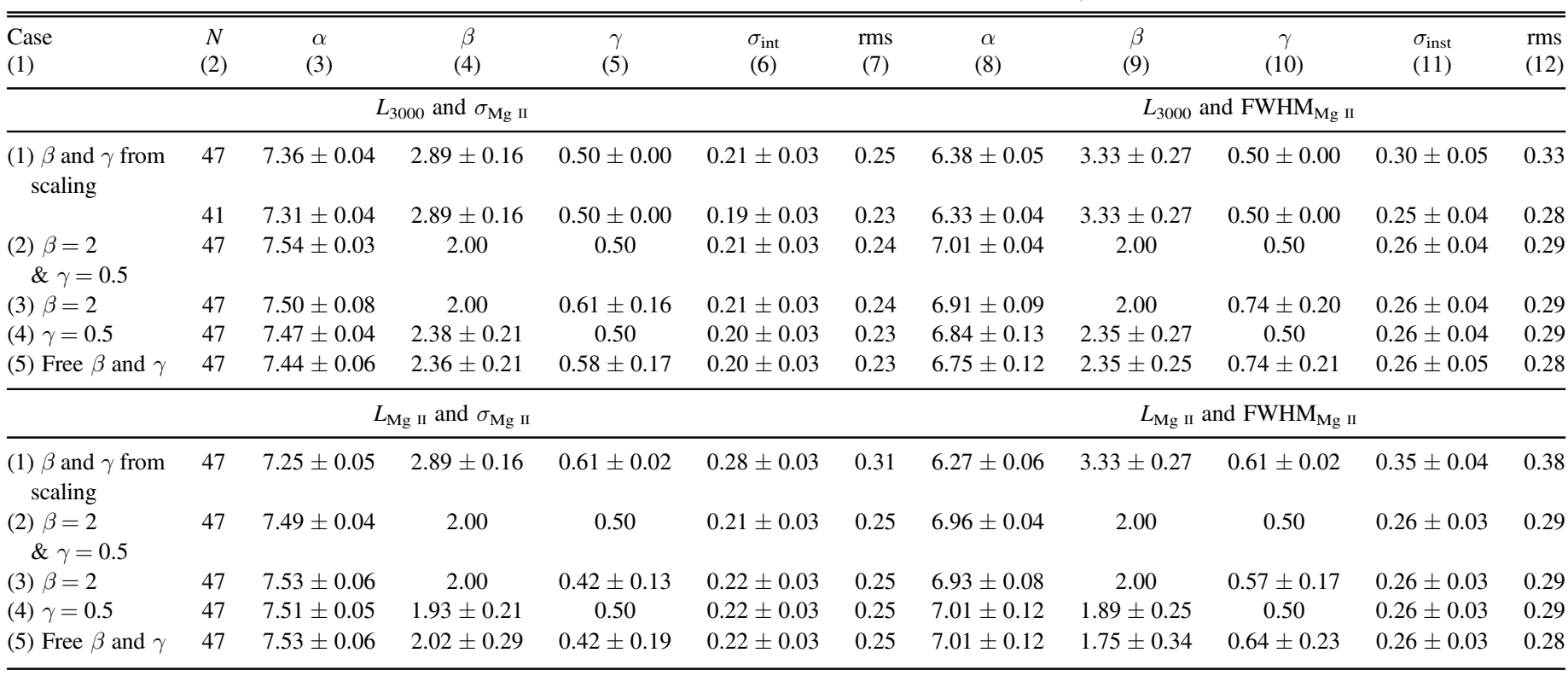

Note. Col. (1): method of calibration. Col. (2): number of data used in the calibration. Col. (3) and (8): $\alpha$ values. Col. (4) and (9): $\beta$ values. Col. (5) and (10): $\gamma$ values. Col. (6) and (11): intrinsic scatter. Col. (7) and (12): rms scatter.

calibration (i.e., based on $\mathrm{Mg}$ II line dispersion and $L_{3000}$ ), the intrinsic scatter between $\mathrm{H} \beta$-based mass and $\mathrm{Mg}$ II-based mass is $\sim 0.17 \mathrm{dex}$, indicating that even with the measurements based on high-quality spectra, the single-epoch mass determined from UV measurements suffers from additional uncertainties by more than 0.17 dex, if we assume the $\mathrm{H} \beta$-based mass represents the true $M_{\mathrm{BH}}$.

As a consistency check, we also performed the same calibration for $\mathrm{Mg}$ II-based masses, using the fiducial mass determined from FWHM of $\mathrm{H} \beta$ and $L_{5100}$ (for issues on the FWHM versus $\sigma$, see Peterson et al. 2004; Collin et al. 2006; Denney et al. 2009; Park et al. 2012b). As presented in Table 4, we obtained slightly worse calibrations with a larger scatter. Again, the line dispersion of $\operatorname{Mg}$ II $\left(\sigma_{\mathrm{Mg} \text { II }}\right)$ and $L_{3000}$ provided the best calibration among all paris of velocity and luminosity measures.

\section{Discussion}

\subsection{Systematic Uncertainties in Mg II-based Mass Estimates}

Although the single-epoch estimators are powerful in determining $M_{\mathrm{BH}}$ of a large sample, the uncertainty of the mass estimates is much more significant than that of the reverberation masses, due to the uncertainty and scatter of the size-luminosity relation (e.g., 0.19 dex reported by Bentz et al. 2013). Moreover, there are additional sources of uncertainties in $\mathrm{Mg}$ II-based mass estimates since these are based on further calibrations of the Mg II line velocity and UV luminosities against $\mathrm{H} \beta$-based $M_{\mathrm{BH}}$ since there is no available $\mathrm{Mg}$ II-based size-luminosity relation.

Here, we discuss several issues to consider in understanding the systematic uncertainties of $\mathrm{Mg}$ II-based mass estimates. 
First, while the variability is the key to measuring the BLR size, it also causes difficulty in calibrating mass estimators. Since for given AGNs with a fixed $M_{\mathrm{BH}}$, luminosity and velocity vary over time, simultaneous observations of the restframe UV and optical are required to properly compare the widths of $\mathrm{H} \beta$ and $\mathrm{Mg}$ II or the UV and optical luminosities. The non-simultaneity often causes difficulties in comparing C IV and $\mathrm{H} \beta$ line widths (see Denney 2012), while it can be avoided by selecting AGNs at optimal redshifts so that both $\mathrm{Mg}$ II and $\mathrm{H} \beta$ lines can be simultaneously obtained in the observed spectral range (e.g., McGill et al. 2008; Wang et al. 2009; Shen et al. 2011).

Instead of the continuum luminosity at $3000 \AA$, the line luminosity of $\mathrm{Mg}$ II can be utilized to determine $M_{\mathrm{BH}}$ by assuming that $\mathrm{Mg}$ II line luminosity varies in response to UV continuum luminosity (for example, see a recent study by Zhu et al. 2017). As we have shown in Figure 7, the scatter increases by a factor of 2 when we compare $L_{5100}$ with $L_{\mathrm{Mg} \text { II }}$ instead of $L_{3000}$. Due to this less tight correlation, the uncertainty of $M_{\mathrm{BH}}$ estimates based on $L_{\mathrm{Mg} \text { II }}$ will be larger than those based on $L_{3000}$ (see the scatter in Figure 9).

Second, the Balmer continuum present in the UV spectral range may cause a systematic uncertainty in measuring UV continuum luminosity. Without a proper fitting and subtraction of the Balmer continuum, $L_{3000}$ may be overestimated, resulting in a higher $M_{\mathrm{BH}}$. However, the systematic effect of the Balmer continuum is limited since its contribution at $3000 \AA$ is relatively small. For example, Kovačević-Dojčinović et al. (2017) reported that $L_{3000}$ changes by $\sim 10 \%$ on average, hence $M_{\mathrm{BH}}$ is overestimated by an average of $\sim 5 \%$.

Third, various studies have reported a nonlinear relationship between $\mathrm{H} \beta$ and $\mathrm{Mg}$ II line widths (Salviander et al. 2007; Wang et al. 2009; Shen et al. 2011). The FWHM of $\mathrm{H} \beta$ is larger than that of $\mathrm{Mg}$ II by more than $20 \%$ for AGNs with a very broad $\mathrm{H} \beta$ line (Marziani et al. 2013) while the line widths of $\mathrm{H} \beta$ and $\mathrm{Mg}$ II are more consistent in AGNs with a narrower $\mathrm{H} \beta$ line. This nonlinear relationship may cast doubts on $\mathrm{Mg}$ IIbased mass estimates since, while $\beta=2$ is used for the $\mathrm{H} \beta$ line in Equation (1), $\beta$ is forced to be larger than 2 for $\mathrm{Mg} \mathrm{II}$, violating the virial assumption. It is not clear whether the $\mathrm{H} \beta$ line width overestimates the true velocity of BLR gas when the FWHM of $\mathrm{H} \beta$ is larger than, for example, $4000 \mathrm{~km} \mathrm{~s}^{-1}$ (see the discussion on Population B in Marziani et al. 2013), or Mg II line width underestimates the velocity of BLR gas. The fact that the line profiles of $\mathrm{Mg}$ II are rather similar to each other, regardless of the width of the line (see Figure 4) may imply that there is a systematic issue in measuring the $\mathrm{H} \beta$ line width, particularly FWHM, when the line is extremely broad. In practice, we performed the calibration with/without the correlation results between $\mathrm{H} \beta$ and $\mathrm{Mg}$ II line widths. It seems better to assume a virial relation (i.e., $\beta=2$ ) since the $\mathrm{Mg}$ II$\mathrm{H} \beta$ width correlation results depend on the sample and dynamic range, suffering from systematic effects.

Fourth, it is not clear whether the narrow component of $\mathrm{Mg}$ II should be separately fitted and subtracted to properly measure the width of the broad component of Mg II. Subtracting the narrow component originating from the narrow-line region is a typical process in fitting and measuring the width of the broad component of $\mathrm{H} \beta$, by assuming the narrow $\mathrm{H} \beta$ profile is identical to other narrow lines, i.e., O III $\lambda 5007$. In the case of $\mathrm{Mg}$ II, however, it is practically difficult to constrain the profile of the narrow component. Thus, most previous studies do not attempt to subtract it. In contrast, Wang et al. (2009) used two components, respectively for the broad and narrow components in $\mathrm{Mg}$ II, and measured the FWHM of the broad component to determine $M_{\mathrm{BH}}$. However, FWHM measurements suffer from significant uncertainties since the peak of the line profile will be strongly dependent on the amount of flux assigned to the narrow component.

In our study, we see no clear sign of the presence of a narrow component in $\mathrm{Mg}$ II even when we see a strong and clear narrow component in $\mathrm{H} \beta$. Note that this can be partly due to the lower spectral resolution in the $\mathrm{Mg}$ II area (i.e., $\sim 145 \mathrm{~km} \mathrm{~s}^{-1}$ ), although a narrow line with a typical velocity dispersion of a few hundred $\mathrm{km} \mathrm{s}^{-1}$ (i.e., in the case of the O III; Woo et al. 2016) can be resolved in our Keck spectra. To test the potential effect of the narrow component in $\mathrm{Mg}$ II, we used the O II line profile to constrain it, assuming the profiles of narrow lines in the narrow-line region (i.e., O II and narrow Mg II) are similar. As Malkan et al. (2017) reported that the typical value of Mg IIto-O II ratio in Seyfert 2 galaxies is $\sim 0.1$, we take the O II line profile, after multiplying by 0.1 , as a narrow component of $\mathrm{Mg}$ II and subtract it from the Mg II line to calculate the line width of the broad component of $\mathrm{Mg}$ II. However, we find that this makes no difference in the line width measurements since $\mathrm{O}$ II is much weaker than $\mathrm{Mg}$ II, hence the narrow component of $\mathrm{Mg}$ II is negligible in most objects. Thus, we did not subtract the potential narrow component and used the total line profile to measure the FWHM and line dispersion. Note that the measurements of line dispersion are not significantly affected by the subtraction or inclusion of the narrow component.

Fifth, we investigated whether the systematic difference between $\mathrm{Mg}$ II- and $\mathrm{H} \beta$-based masses shows any dependency on other AGN parameters, i.e., Eddington ratio, FWHM of $\mathrm{H} \beta$, or the systematic difference of the line profiles between $\mathrm{Mg}$ II and $\mathrm{H} \beta$ in Figure 10 (top panels). We also checked whether the systematic difference of $M_{\mathrm{BH}}$ is due to the eigenvector 1 by calculating the flux ratio between $\mathrm{O}$ III and Fe II, which is integrated in the spectral range and the spectral slope 4434-4684 $\AA$ (e.g., see Woo et al. 2015), and the flux ratio between $\mathrm{O}$ III and the narrow component of $\mathrm{H} \beta$ (bottom panel in Figure 10). For the first five parameters, we found no significant trend, suggesting that Mg II-based masses are not significantly biased due to the Eddington ratio, line width, or the Fe II strength.

In contrast, we expect to see a broad trend between the UVto-optical mass ratio and the UV-to-optical luminosity ratio (i.e., $\left.L_{3000} / L_{5100}\right)$ since single-epoch $M_{\mathrm{BH}}$ correlates with continuum luminosity as far as the size-luminosity relation (i.e., $M_{\mathrm{BH}} \propto \mathrm{L}^{0.5}$ ) is used for determining $M_{\mathrm{BH}}$. For given $L_{5100}$ and $\mathrm{H} \beta$-based mass, for example, if the spectral slope becomes steeper (i.e., higher $L_{3000} / L_{5100}$ ratio), then $L_{3000}$, and consequently, Mg II-based mass will be systematically higher. We see this trend in Figure 10. To correct for it, we obtain the best-fit slope of $1.02 \pm 0.18$ and the intercept of $0.17 \pm 0.05$ (last panel in Figure 10), and add the following color correction term to Equation (1):

$$
\Delta C=-1.02 \times \log \left(L_{3000} / L_{5100}\right)+0.17 .
$$

Since $L_{5100}$ will not be available for $M_{\mathrm{BH}}$ determination for high- $z$ AGNs, we can instead use the spectral slope $\alpha_{\lambda}$, with which we model the local UV/optical AGN continuum as a power law, $f_{\lambda} \propto \lambda^{\alpha_{\lambda}}$. Using $L_{3000} / L_{5100}=3000 f_{3000} / 5100$ $f_{5100}=(3000 / 5100)^{1+\alpha_{\lambda}}$, we derive the correction term as a 

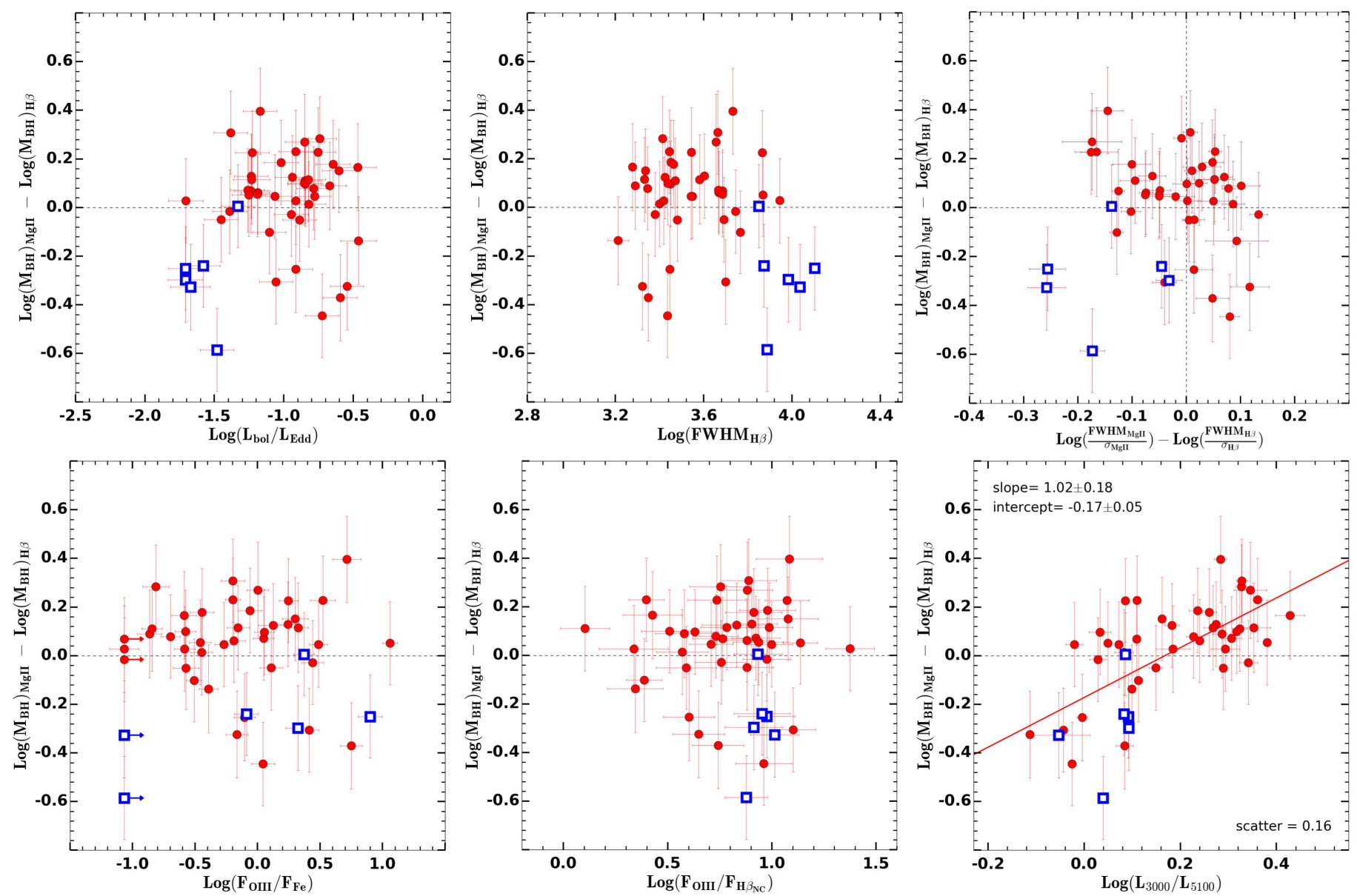

Figure 10. Testing systematic trends with Eddington ratio (top left), $\mathrm{H} \beta$ FWHM (top middle), the difference of line profiles between $\mathrm{H} \beta$ and $\mathrm{Mg}$ II (top right), $F_{\mathrm{O} \text { III }} / F_{\mathrm{Fe} \text { II }}$ (bottom left), $F_{\mathrm{O} \text { III }} / F_{\mathrm{H} \beta \text {,narrow }}$ (bottom middle), $L_{3000} / L_{5100}$ (bottom right). Open blue squares show the six AGNs, S23, S24, W02, W03, W12, and W20, with very different line profiles between $\mathrm{Mg}$ II and $\mathrm{H} \beta$.

function of $\alpha_{\lambda}$ :

$$
\Delta C=0.24\left(1+\alpha_{\lambda}\right)+0.17
$$

Note that the mean $\alpha_{\lambda}$ of the Keck sample is $-1.73 \pm 0.60$ (i.e., $\alpha_{\nu}=-0.27 \pm 0.60$ ), which is slightly bluer than the average spectral slope $\alpha_{\nu}=-0.44$ of the SDSS quasars (Vanden Berk et al. 2001). Once applied, this correction term will reduce the systematic uncertainty of $\mathrm{Mg}$ II-based masses due to the large range of the spectral slope between the UV and optical wavelength range. However, the correction is relatively small. For example, if the spectral slope changes from $\alpha_{\lambda}=-1.5$ to $\alpha_{\lambda}=-2$, the correction on $M_{\mathrm{BH}}$ is $\sim 0.1$ dex. Thus, if the spectral slope is difficult to determine due to the low $\mathrm{S} / \mathrm{N}$, strong Fe II blends, limited spectral range, or internal dust extinction, this correction can be ignored.

\subsection{Comparison of Various Mg II-based Mass Estimators}

There have been various calibrations of $M_{\mathrm{BH}}$ estimators based on $\mathrm{Mg}$ II in the literature, and here we investigate how $M_{\mathrm{BH}}$ changes depending on the choice of the estimators. In Figure 11, we present the calculated $M_{\mathrm{BH}}$ for given pairs of $\mathrm{Mg}$ II line width and $L_{3000}$ based on several UV mass estimators. From our calibrations, we choose Case 2 as the best calibration, and Case 5 as an extreme calibration. In the case of $M_{\mathrm{BH}}$ based on Mg II line dispersion, McGill et al.
(2008) reported the mass calibrators, and we compare our estimators with theirs in the top panels. Case 2 with the fixed $\beta$ and $\gamma$ provides a similar $M_{\mathrm{BH}}$ compared to McGill et al. (2008), with a systematic offset by $0.2-0.3 \mathrm{dex}$. The difference in the normalization is mainly due to the change of the width measurements. Since for given objects, Mg II line dispersion becomes smaller due to the Tsuzuki et al. (2006) template that we used in our analysis, while McGill et al. (2008) used the Fe II template of Vestergaard \& Wilkes (2001). Thus, $\alpha$ becomes larger for UV-based $M_{\mathrm{BH}}$ to be consistent with given $\mathrm{H} \beta$-based mass.

In the case of FWHM, we compare our estimator with those of McGill et al. (2008), Wang et al. (2009), and Shen et al. (2011). Compared to Case 2 (red in Figure 11), other estimators derive somewhat lower $M_{\mathrm{BH}}$, and the equi-mass line is steeper because of $\beta$ lower than 2 or $\gamma$ higher than 0.5. Note that, depending on the choice of the estimators, $M_{\mathrm{BH}}$ will be systematically larger or smaller for AGNs with very broad lines (or lower luminosity). These results indicate that the inferred shape of the mass function of high- $z$ AGNs will be sensitive to the choice of UV $M_{\mathrm{BH}}$ estimator. Note that for estimating $M_{\mathrm{BH}}$ using large survey data, FWHM of Mg II, rather than line dispersion, is often used since the spectral quality in survey data is not sufficient to measure the line dispersion of broad lines. Thus, a careful interpretation is required to understand the mass distribution and mass function of high- $z$ AGNs. 

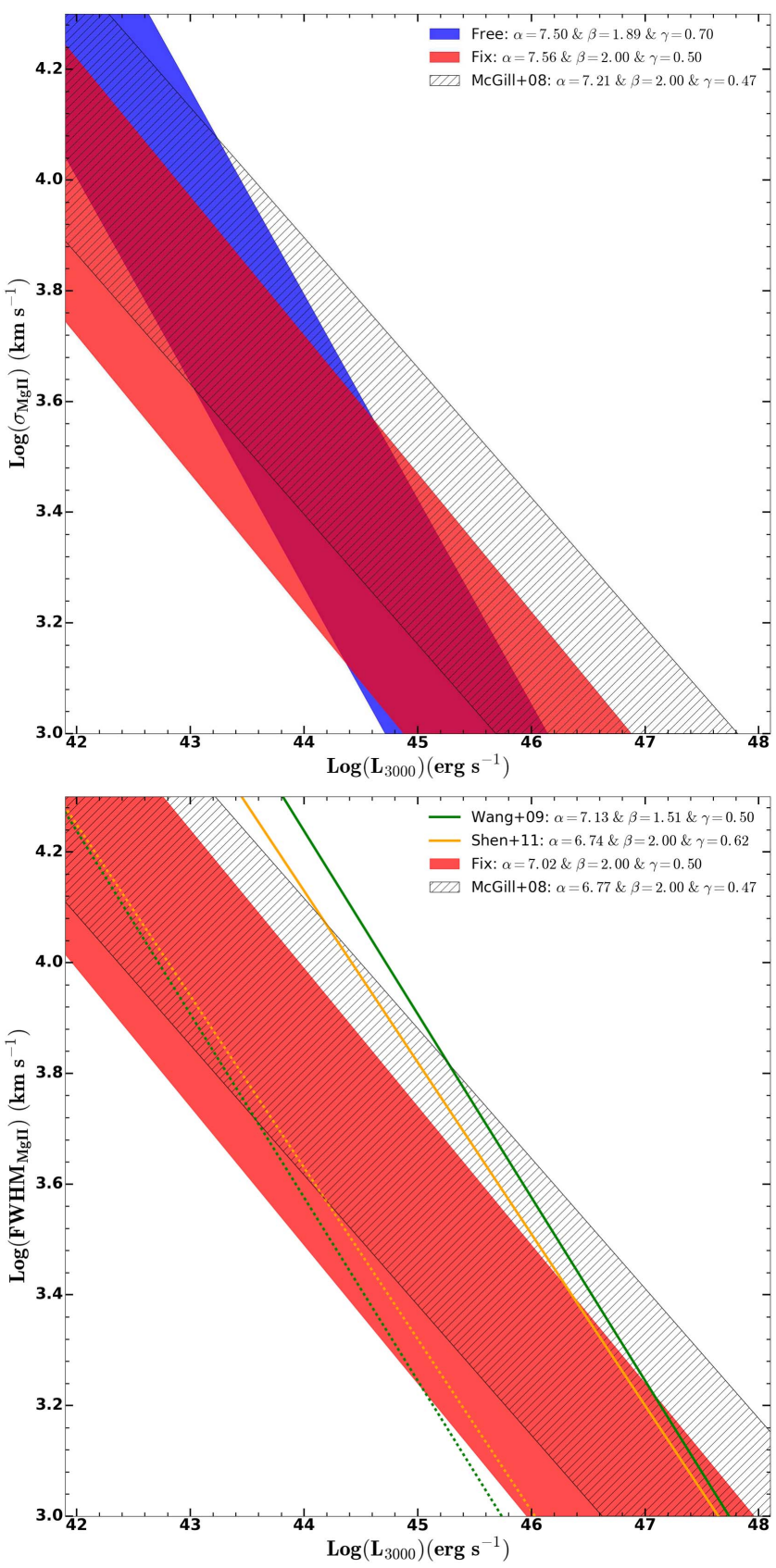

Figure 11. Comparison of $M_{\mathrm{BH}}$ estimates for given pairs of velocity and luminosity measures depending on the choice of UV mass estimator. Top: $M_{\mathrm{BH}}$ based on $\mathrm{Mg}$ II line dispersion from our estimators with fixed $\beta=2 \& \gamma=0.5$ (red area) or free fit results (blue area), compared to that of McGill et al. (2008) (hatched area). For each mass estimator, the area is defined by two equi-mass lines at $\mathrm{M}_{\mathrm{BH}} \overline{1} 0^{8}$ and $\mathrm{M}_{\mathrm{BH}} \overline{1} 0^{9}$, showing the systematic difference among various mass estimators. Bottom: $M_{\mathrm{BH}}$ based on Mg II FWHM compared to those of Wang et al. (2009) (green line) and Shen et al. (2011) (orange line).

\section{Conclusions}

In this paper, we present a new calibration of $M_{\mathrm{BH}}$ estimators, using a sample of $52 \mathrm{AGNs}$ at $z \sim 0.36$ and $z \sim 0.52$ over the $M_{\mathrm{BH}}$ range $7.4<\log M_{\mathrm{BH}}<9$, for which high-quality Keck spectra are available to properly measure line widths and UV and optical luminosities. In addition, we utilize the measurements of SDSS AGNs from literature to increase the dynamic range. The main results are summarized as follows.
(1) There are a number of AGNs for which $\mathrm{H} \beta$ is much broader than $\mathrm{Mg}$ II, particularly for AGNs with a large $\mathrm{H} \beta$ FWHM (see also Wang et al. 2009; Marziani et al. 2013). Consequently, we obtain a sub-linear relationship between $\mathrm{Mg}$ II and $\mathrm{H} \beta$ both for FWHM and line dispersion.

(2) By comparing optical continuum luminosity at $5100 \AA$ with UV continuum luminosity and $\mathrm{H} \beta$ line luminosity, we find a correlation close to a linear relationship, while the relation with $\mathrm{Mg}$ II line luminosity is somewhat sub-linear, reflecting the Baldwin effect in the UV.

(3) We perform a cross-calibration of $M_{\mathrm{BH}}$ estimators using various combinations of velocity and luminosity indicators measured from the rest-frame UV and optical spectra, using the the mass based on $\mathrm{H} \beta$ line dispersion and L5100 as a reference mass. $M_{\mathrm{BH}}$ from the new calibrations using $\mathrm{H} \beta$ line and optical luminosities are consistent with each other with an intrinsic scatter less than 0.1 dex and a rms scatter of $\sim 0.1$ dex.

(4) In the case of UV mass estimators based on the Mg II line, a comparison with the reference $\mathrm{H} \beta$-based masses shows an intrinsic scatter of $0.17-0.28 \mathrm{dex}$ and a rms scatter of $\sim 0.2-0.3$ dex, suggesting that there is an additional uncertainty larger than $\sim 0.2$ dex, depending on the choice of line width (i.e., line dispersion or FWHM) and luminosity measures (i.e., $L_{3000}$ or $L_{\mathrm{Mg} \text { II }}$ ). Over all, we find that the pair of $\mathrm{Mg}$ II line dispersion and $L_{3000}$ provides the best calibration with an additional $0.17 \pm 0.03$ dex uncertainty. In the case of $\mathrm{H} \beta$ single-epoch mass estimates, the uncertainties are mainly introduced by three sources. First, the uncertainty of the virial factor, which is $0.12-0.15$ dex based on the comparison of the $M_{\mathrm{BH}^{-}} \sigma_{*}$ relation between the reverberation-mapped AGNs and quiescent galaxies (Woo et al. 2010, 2015), or 0.4 dex based on the dynamical modeling of the five reverberation-mapped AGNs with velocity-resolved measurements (Pancoast et al. 2014). Second, the scatter in the $\mathrm{H} \beta$ size-luminosity relation is $0.13-0.19$ dex, depending on the choice of more reliable measurements (Bentz et al. 2013). Third, the random variability of the line width and luminosity introduces $\sim 0.1 \mathrm{dex}$ scatter (Park et al. 2012b). Compared to the total uncertainty of $\mathrm{H} \beta$-based mass estimates, which can be $0.3-0.4$ dex, the additional $0.17-0.28$ dex uncertainty from the calibration of UV mass estimators is somewhat smaller. However, the overall uncertainty of $\mathrm{Mg}$ II-based mass is larger than that of $\mathrm{H} \beta$ masses.

In this paper, we calibrated UV and optical $M_{\mathrm{BH}}$ estimators based on single-epoch measurements. While future direct measurements of the $\mathrm{Mg}$ II time lag for a sizable sample of AGNs will enable us to reduce the systematic uncertainties in the single-epoch mass estimates, the updated and calibrated $\mathrm{Mg}$ II mass estimators in this paper will be useful for revisiting $M_{\mathrm{BH}}$-related issues for high- $z$ AGNs.

This work has been supported by the Basic Science Research Program through the National Research Foundation of Korea government (2016R1A2B3011457 and No. 2017R1A5A1070354). We thank the anonymous referee for useful suggestions.

\section{ORCID iDs}

Jong-Hak Woo (ib https://orcid.org/0000-0002-8055-5465 Marios Karouzos (iD https://orcid.org/0000-0002-8858-3188 Daeseong Park (D) https://orcid.org/0000-0001-9877-1732 Matthew A. Malkan (i) https://orcid.org/0000-00016919-1237 
Tommaso Treu (10) https://orcid.org/0000-0002-8460-0390

Vardha N. Bennert (1) https://orcid.org/0000-0003-2064-0518

\section{References}

Baldwin, J. A. 1977, ApJ, 214, 679

Barth, A. J., Bennert, V. N., Canalizo, G., et al. 2015, ApJS, 217, 26 Barth, A. J., Pancoast, A., Thorman, S. J., et al. 2011, ApJL, 743, L4 Bennert, V. N., Treu, T., Woo, J.-H., et al. 2010, ApJ, 708, 1507

Bentz, M. C., Denney, K. D., Grier, C. J., et al. 2013, ApJ, 767, 149

Bentz, M. C., Peterson, B. M., Netzer, H., Pogge, R. W., \& Vestergaard, M. 2009, ApJ, 697, 160

Bentz, M. C., Peterson, B. M., Pogge, R. W., Vestergaard, M., \& Onken, C. A. 2006, ApJ, 644, 133

Blandford, R. D., \& McKee, C. F. 1982, ApJ, 255, 419

Boroson, T. A., \& Green, R. F. 1992, ApJS, 80, 109

Bruzual, G., \& Charlot, S. 2003, MNRAS, 344, 1000

Cackett, E. M., Gültekin, K., Bentz, M. C., et al. 2015, ApJ, 810, 86

Collin, S., Kawaguchi, T., Peterson, B. M., \& Vestergaard, M. 2006, A\&A, 456, 75

Denney, K. D. 2012, ApJ, 759, 44

Denney, K. D., Peterson, B. M., Dietrich, M., Vestergaard, M., \& Bentz, M. C. 2009, ApJ, 692, 246

Dong, X.-B., Wang, T.-G., Wang, J.-G., et al. 2009, ApJL, 703, L1

Fausnaugh, M. M. 2017, PASP, 129, 024007

Greene, J. E., \& Ho, L. C. 2005, ApJ, 630, 122

Grier, C. J., Martini, P., Watson, L. C., et al. 2013, ApJ, 773, 90

Karouzos, M., Woo, J.-H., Matsuoka, K., et al. 2015, ApJ, 815, 128

Kaspi, S., Maoz, D., Netzer, H., et al. 2005, ApJ, 629, 61

Kaspi, S., Smith, P. S., Netzer, H., et al. 2000, ApJ, 533, 631

Kormendy, J., \& Ho, L. C. 2013, ARA\&A, 51, 511

Kovačević-Dojčinović, J., Marčeta-Mandić, S., \& Popović, L. Č. 2017, arXiv:1707.08251

Malkan, M. A., Jensen, L. D., Rodriguez, D. R., Spinoglio, L., \& Rush, B. 2017, ApJ, 846, 102

Malkan, M. A., \& Sargent, W. L. W. 1982, ApJ, 254, 22
Markwardt, C. B. 2009, in Astronomical Data Analysis Software and Systems XVIII, ed. D. A. Bohlender, D. Durand, \& P. Dowler (San Francisco, CA: ASP), 251

Marziani, P., Sulentic, J. W., Plauchu-Frayn, I., \& del Olmo, A. 2013, A\&A, 555, A89

McGill, K. L., Woo, J.-H., Treu, T., \& Malkan, M. A. 2008, ApJ, 673, 703

McLure, R. J., \& Dunlop, J. S. 2004, MNRAS, 352, 1390

McLure, R. J., \& Jarvis, M. J. 2002, MNRAS, 337, 109

Metzroth, K. G., Onken, C. A., \& Peterson, B. M. 2006, ApJ, 647, 901

Oke, J. B., Cohen, J. G., Carr, M., et al. 1995, PASP, 107, 375

Pancoast, A., Brewer, B. J., Treu, T., et al. 2014, MNRAS, 445, 3073

Park, D., Kelly, B. C., Woo, J.-H., \& Treu, T. 2012a, ApJ, 203, 6

Park, D., Woo, J.-H., Bennert, V., et al. 2015, ApJ, 799, 164

Park, D., Woo, J.-H., Treu, T., et al. 2012b, ApJ, 747, 30

Park, S., Woo, J.-H., Romero-Colmenero, E., et al. 2017, ApJ, 847, 125

Peterson, B. M. 1993, PASP, 105, 247

Peterson, B. M., Ferrarese, L., Gilbert, K. M., et al. 2004, ApJ, 613, 682

Reichert, G. A., Rodriguez-Pascual, P. M., Alloin, D., et al. 1994, ApJ, 425, 582

Salviander, S., Shields, G. A., Gebhardt, K., \& Bonning, E. W. 2007, ApJ, 662, 131

Schlegel, D. J., Finkbeiner, D. P., \& Davis, M. 1998, ApJ, 500, 525

Shen, Y., Horne, K., Grier, C. J., et al. 2016, ApJ, 818, 30

Shen, Y., Richards, G. T., Strauss, M. A., et al. 2011, ApJS, 194, 45

Treu, T., Malkan, M. A., \& Blandford, R. D. 2004, ApJL, 615, L97

Tsuzuki, Y., Kawara, K., Yoshii, Y., et al. 2006, ApJ, 650, 57

Vanden Berk, D. E., Richards, G. T., Bauer, A., et al. 2001, AJ, 122, 549

Vestergaard, M., \& Peterson, B. M. 2006, ApJ, 641, 689

Vestergaard, M., \& Wilkes, B. J. 2001, ApJS, 134, 1

Wandel, A., Peterson, B. M., \& Malkan, M. A. 1999, ApJ, 526, 579

Wang, J.-G., Dong, X.-B., Wang, T.-G., et al. 2009, ApJ, 707, 1334

Woo, J.-H. 2008, AJ, 135, 1849

Woo, J.-H., Bae, H.-J., Son, D., \& Karouzos, M. 2016, ApJ, 817, 108

Woo, J.-H., Schulze, A., Park, D., et al. 2013, ApJ, 772, 49

Woo, J.-H., Treu, T., Barth, A. J., et al. 2010, ApJ, 716, 269

Woo, J.-H., Treu, T., Malkan, M. A., \& Blandford, R. D. 2006, ApJ, 645, 900

Woo, J.-H., \& Urry, C. M. 2002, ApJ, 579, 530

Woo, J.-H., Yoon, Y., Park, S., et al. 2015, ApJ, 801, 38

Zheng, W., \& Malkan, M. A. 1993, ApJ, 415, 517

Zhu, D., Sun, M., \& Wang, T. 2017, ApJ, 843, 30 